\title{
Dynamics of stochastic Boissonade system on the time-varying domain
}

\section{Zhen Zhang and Jianhua Huang*}

\section{${ }^{*}$ Correspondence:}

jhhuang32@nudt.edu.cn

College of Science, National

University of Defense Technology,

Changsha, 410073, P.R. China

\begin{abstract}
The current paper is devoted to studying the stochastic Boissonade system defined on time-varying domains. The existence and uniqueness of strong and weak solutions for the stochastic Boissonade system are established. Moreover, the existence of pullback attractor for the 'partial-random' system generated by the weak solution is also presented.
\end{abstract}

MSC: 35K57; 35K90; 37L30

Keywords: pullback attractor; partial-random dynamical systems; stochastic Boissonade system; time-varying domain

\section{Introduction}

The well-posedness and dynamics of the partial differential equations defined on the timevarying domains are interesting questions to study, and they have attracted a lot of attentions recently. There are many papers on this topic, we refer the reader to [1-10] and the references therein. The stochastic dynamical systems defined on time-varying domains are more attractive. Crauel, Kloeden, and Real established the framework for deterministic PDE on time-varying domains, and later, they also developed a new approach to defined noise on time-varying domain, and established the existence and uniqueness of the solutions for stochastic partial different equations with additive noise on time-varying domains in [11]. Recently, Crauel, Kloeden, and Yang developed the theory of 'partialrandom' dynamical systems to obtain the existence of random attractors for stochastic reaction-diffusion equations on time-varying domains in [4].

Reaction-diffusion systems are usually used to describe the Turing pattern in a class of chemical or biological systems, and the Turing pattern was observed in the chloriteiodine-malonic acid reaction in 1992. Dufiet and Boissonade in [12] were first to introduce the following reaction-diffusion systems (we called it a Boissonade system):

$$
\left\{\begin{array}{l}
\frac{\partial u}{\partial t}=d_{1} \Delta u+u-\alpha v+\gamma u v-u^{3}, \\
\frac{\partial v}{\partial t}=d_{2} \Delta v+u-\beta v,
\end{array}\right.
$$

to exhibit the Turing pattern of the model to describe the relation between the genuine homogeneous $2 D$ systems and the $3 D$ monolayers, where $d_{1}, d_{2}, \alpha, \gamma$, and $\beta$ are positive constants.

(c) 2016 Zhang and Huang. This article is distributed under the terms of the Creative Commons Attribution 4.0 International License (http://creativecommons.org/licenses/by/4.0/), which permits unrestricted use, distribution, and reproduction in any medium, provided you give appropriate credit to the original author(s) and the source, provide a link to the Creative Commons license, and indicate if changes were made. 
The Boissonade system (1.1) is quite different from the Fitzhugh-Nagumo system in [13] and [14], the square term $u^{2}$ in the Fitzhugh-Nagumo system is replaced by the cross term $u v$, leading to the nonlinearity of the second equation in the Boissonade system, and it induces more difficulties to obtain the uniqueness of the solution. Recently, Tu in [15] proved the existence of the global attractor for the Boissonade system (1.1). Due to the time-varying domain, the stochastic partial differential equation induces the new partial random dynamical systems, which is very interesting, we refer the reader to [11] for more details.

Motivated by the idea of Crauel, Kloeden, and Real in [3] and Crauel, Kloeden, and Yang in [11], we study the stochastic Boissonade system (SBS) on the time-varying domain by using some tricks derived from the Sobolev embedding theorem to obtain a unique solution for the SBS, and we establish the existence of a pullback attractor for the 'partial-random' dynamical system generated by the weak solution for the stochastic Boissonade system on the time-varying domain.

The rest of the paper is arranged as follows. In Section 2, some notations on time-varying domains are introduced. Sections 3 and 4 are devoted to proving the existence and uniqueness of solutions of random equations defined on fixed domains which are transformed from time-varying domains. The existence of the pullback attractor for the process generalized by the weak solution is presented in Section 5 .

\section{SBS defined on time-varying domains}

In this section, we will introduce some notions and functional spaces on time-varying domains, following [11], and derive the Boissonade system with additive noise on the timevarying domain.

\subsection{Assumption on the time-varying domain}

Let $\mathcal{O}$ be a nonempty bounded open subset of $\mathbb{R}^{N}$ with $C^{2}$ boundary $\partial \mathcal{O}$, and $r=r(y, t)$ a vector function

$$
r \in C^{1}\left(\overline{\mathcal{O}} \times \mathbb{R} ; \mathbb{R}^{N}\right)
$$

such that

$$
r(\cdot, t): \mathcal{O} \rightarrow \mathcal{O}_{t} \quad \text { is a } C^{2} \text {-diffeomorphism for all } t \in \mathbb{R}
$$

$\bar{r}(\cdot, t)=r^{-1}(\cdot, t)$ is the inverse of $r(\cdot, t)$ satisfying the property

$$
\bar{r} \in C^{2,1}\left(\bar{Q}_{\tau, T} ; \mathbb{R}^{N}\right) \text { for all } \tau<T,
$$

i.e., $\bar{r}, \frac{\partial \bar{r}}{\partial t}, \frac{\partial \bar{r}}{\partial x_{i}}$ and $\frac{\partial^{2} \bar{r}}{\partial x_{i} \partial x_{j}}$ belong to $C\left(\bar{Q}_{\tau, T} ; \mathbb{R}^{N}\right)$ for all $1 \leq i, j \leq N$ and for any $\tau<T$. Then $\left\{\mathcal{O}_{t}\right\}_{t \in[\tau, T]}$ is a family of nonempty bounded open subsets of $\mathbb{R}^{N}(N \leq 3)$.

Define

$$
\begin{aligned}
& Q_{\tau, T}:=\bigcup_{t \in(\tau, T)} \mathcal{O}_{t} \times\{t\} \quad \text { for all } T>\tau, \\
& Q_{\tau}:=\bigcup_{t \in(\tau, \infty)} \mathcal{O}_{t} \times\{t\} \quad \text { for all } \tau \in \mathbb{R},
\end{aligned}
$$




$$
\Sigma_{\tau, T}:=\bigcup_{t \in(\tau, T)} \partial \mathcal{O}_{t} \times\{t\} \quad \text { for all } T>\tau
$$

and

$$
\Sigma_{\tau}:=\bigcup_{t \in(\tau, \infty)} \partial \mathcal{O}_{t} \times\{t\} \quad \text { for all } \tau \in \mathbb{R}
$$

For any $T>\tau$, the set $Q_{\tau, T}$ is an open subset of $\mathbb{R}^{N+1}$ with the boundary

$$
\partial Q_{\tau, T}:=\Sigma_{\tau, T} \cup\left(\partial \mathcal{O}_{\tau} \times\{\tau\}\right) \cup\left(\partial \mathcal{O}_{T} \times\{T\}\right)
$$

\subsection{Assumption on noise}

Assume that $(\Omega, \mathcal{F}, \mathbb{P})$ be a probability space, a sequence $\left\{w_{j}(t): t \in[0, \infty)\right\}_{j \geq 1}$ of mutually independent two-sided standard scalar Wiener processes adapted to a common filtration $\left\{\mathcal{F}_{t}: t \in[0, \infty)\right\}$ in $\mathcal{F}$. Let $\left\{\phi_{j}\right\}_{j \geq 1} \subset H_{0}^{1}(\mathcal{O}) \subset L^{2}(\mathcal{O})$ and $\left\{\varphi_{j}\right\}_{j \geq 1} \subset H_{0}^{1}(\mathcal{O}) \subset L^{2}(\mathcal{O})$ be two sequences of functions such that

$$
\sum_{j=1}^{\infty}\left\|\phi_{j}\right\|_{H_{0}^{1}(\mathcal{O})} \leq \infty, \quad \sum_{j=1}^{\infty}\left\|\varphi_{j}\right\|_{H_{0}^{1}(\mathcal{O})} \leq \infty .
$$

Define

$$
\Phi_{j}:=\phi_{j}(\bar{r}(x, t)), \quad \Psi_{j}:=\varphi_{j}(\bar{r}(x, t)), \quad x \in \mathcal{O}_{t}, t \in[0, \infty), j=1,2, \ldots
$$

It follows from [7] that, for all $t \in \mathbb{R}$,

$$
\sum_{j=1}^{\infty}\left\|\Phi_{j}\right\|_{H_{0}^{1}\left(\mathcal{O}_{t}\right)} \leq \infty, \quad \sum_{j=1}^{\infty}\left\|\Psi_{j}\right\|_{H_{0}^{1}\left(\mathcal{O}_{t}\right)} \leq \infty
$$

Consider the $L^{2}\left(\mathcal{O}_{t}\right)$-valued $\mathcal{F}_{t}$-adapted stochastic processes. Define

$$
M_{1}:=\sum_{j=1}^{\infty} \Phi_{j}(t) w_{j}(t), \quad M_{2}:=\sum_{j=1}^{\infty} \Psi_{j}(t) w_{j}(t), \quad t \geq 0 .
$$

Let $\mathbb{E}$ be the expectation with respect the probability $\mathbb{P}$. Due to the pairwise independence of the $w_{j}(t)$, we have

$$
E\left\|\sum_{j=n}^{m} \Phi_{j}(t) w_{j}(t)\right\|_{L^{2}\left(\mathcal{O}_{t}\right)}^{2}=t \sum_{j=n}^{m}\left\|\Phi_{j}(t)\right\|_{L^{2}\left(\mathcal{O}_{t}\right)}^{2}
$$

and

$$
E\left\|\sum_{j=n}^{m} \Psi_{j}(t) w_{j}(t)\right\|_{L^{2}\left(\mathcal{O}_{t}\right)}^{2}=t \sum_{j=n}^{m}\left\|\Psi_{j}(t)\right\|_{L^{2}\left(\mathcal{O}_{t}\right)}^{2}
$$


for any $t \geq 0, m>n \geq 1$. Therefore, we get $M_{1}(t), M_{2}(t) \in L^{2}\left(\mathcal{O}_{t} \times \Omega\right)$ which are $\mathcal{F}_{t}$-measurable. Then $\left\{M_{1}(t): t \geq 0\right\}$ and $\left\{M_{2}(t): t \geq 0\right\}$ can be viewed as $\mathcal{F}_{t}$-adapted processes with values in $L^{2}\left(\mathcal{O}_{t}\right)$.

Direct computation implies that $\mathbb{E} M_{1}(t)=\mathbb{E} M_{2}(t)=0$,

$$
\mathbb{E}\left\|M_{1}(t)\right\|_{L^{2}\left(\mathcal{O}_{t}\right)}^{2}=t \sum_{j=1}^{\infty}\|\Phi(t)\|_{L^{2}\left(\mathcal{O}_{t}\right)}^{2} \leq t C_{r, t} \sum_{j=1}^{\infty}\|\phi(t)\|_{L^{2}(\mathcal{O})}^{2}
$$

and

$$
\mathbb{E}\left\|M_{2}(t)\right\|_{L^{2}\left(\mathcal{O}_{t}\right)}^{2}=t \sum_{j=1}^{\infty}\|\Psi(t)\|_{L^{2}\left(\mathcal{O}_{t}\right)}^{2} \leq t C_{r, t} \sum_{j=1}^{\infty}\|\varphi(t)\|_{L^{2}(\mathcal{O})}^{2}
$$

for any $t \in[0, \infty)$, where $C_{r, t}=\max _{y \in \overline{\mathcal{O}}} \mathrm{Jac}(r, y, t)$ and $\operatorname{Jac}(r, y, t)$ denoted the absolute value of the determinant of the Jacobi matrix $\left(\frac{\partial r_{i}}{\partial y_{j}}(y, t)\right)_{N \times N}$.

\subsection{Stochastic Boissonade system on the time-varying domain}

Following the arguments in [11], we can study the stochastic Boissonade system with additive noise and homogeneous Dirichlet boundary condition on the time-varying domain as follows:

$$
\left\{\begin{array}{l}
d u=\left(d_{1} \Delta u+u-\alpha v+\gamma u v-u^{3}\right) d t+d M_{1}, \quad \text { in } Q_{0}, \\
d v=\left(d_{2} \Delta v+u-\beta v\right) d t+d M_{2}, \quad \text { in } Q_{0}, \\
u=0, \quad v=0, \quad \text { on } \Sigma_{0}, \\
u(0, x)=u_{0}(x), \quad v(0, x)=v_{0}(x), \quad x \in \mathcal{O}_{0},
\end{array}\right.
$$

where $d M_{1}$ and $d M_{2}$ can be represented by

$$
d M_{1}(t)=\sum_{j=1}^{\infty} \phi_{j}(\bar{r}(x, t)) d w_{j}(t)+\sum_{j=1}^{\infty} w_{j}(t) \nabla_{y} \phi_{j}(\bar{r}(x, t)) \cdot \frac{\partial \bar{r}}{\partial t}(x, t) d t
$$

and

$$
d M_{2}(t)=\sum_{j=1}^{\infty} \varphi_{j}(\bar{r}(x, t)) d w_{j}(t)+\sum_{j=1}^{\infty} w_{j}(t) \nabla_{y} \varphi_{j}(\bar{r}(x, t)) \cdot \frac{\partial \bar{r}}{\partial t}(x, t) d t .
$$

Denote

$$
U(y, t)=u(r(y, t), t), \quad V(y, t)=v(r(y, t), t) \quad \text { for } y \in \mathcal{O}, t \geq 0,
$$

and

$$
a_{j k}(y, t)=\sum_{i=1}^{N} \frac{\partial \bar{r}_{k}}{\partial x_{i}}(r(y, t), t) \frac{\partial \bar{r}_{j}}{\partial x_{i}}(r(y, t), t), \quad j, k=1, \ldots, N
$$


Define $b(y, t)=\left(b_{1}(y, t), \ldots, b_{N}(y, t)\right) \in \mathbb{R}^{N}$ and $c(y, t)=\left(c_{1}(y, t), \ldots, c_{N}(y, t)\right) \in \mathbb{R}^{N}$ by

$$
\begin{aligned}
& b_{k}(y, t)=d_{1} \Delta_{x} \bar{r}_{k}(r(y, t), t)-\frac{\partial \bar{r}_{k}}{\partial t}(r(y, t), t)-d_{1} \sum_{j=1}^{N} \frac{\partial a_{j k}}{\partial y_{j}}(y, t), \quad k=1, \ldots, N, \\
& c_{k}(y, t)=d_{2} \Delta_{x} \bar{r}_{k}(r(y, t), t)-\frac{\partial \bar{r}_{k}}{\partial t}(r(y, t), t)-d_{2} \sum_{j=1}^{N} \frac{\partial a_{j k}}{\partial y_{j}}(y, t), \quad k=1, \ldots, N .
\end{aligned}
$$

Then equations (2.7) on time-varying domains can be rewritten into the following equations on $\mathcal{O} \times[0, \infty)$ :

$$
\left\{\begin{aligned}
d U= & {\left[d_{1} \sum_{j, k=1}^{N} \frac{\partial}{\partial y_{j}}\left(a_{j k} U_{y_{k}}\right)+b \cdot \nabla_{y} U\right.} \\
& \left.+U-\alpha V+\gamma U V-U^{3}+R_{1}\right] d t+d W_{1}, \quad \text { in } \mathcal{O} \times[0, \infty), \\
d V= & {\left[d_{2} \sum_{j, k=1}^{N} \frac{\partial}{\partial y_{j}}\left(a_{j k} V_{y_{k}}\right)+c \cdot \nabla_{y} V\right.} \\
& \left.+U-\beta V+R_{2}\right] d t+d W_{2}, \quad \text { in } \mathcal{O} \times[0, \infty), \\
U=0, \quad V=0, \quad \text { on } \partial \mathcal{O} \times[0, \infty), & \quad V(y, 0)=v(r(y, 0)), \quad y \in \mathcal{O},
\end{aligned}\right.
$$

where

$$
\begin{aligned}
& R_{1}(y, t)=\sum_{j=1}^{\infty} w_{j}(t) \nabla_{y} \phi_{j}(y) \cdot \frac{\partial \bar{r}}{\partial t}(r(y, t), t), \\
& R_{2}(y, t)=\sum_{j=1}^{\infty} w_{j}(t) \nabla_{y} \varphi_{j}(y) \cdot \frac{\partial \bar{r}}{\partial t}(r(y, t), t),
\end{aligned}
$$

and

$$
W_{1}(y, t)=\sum_{j=1}^{\infty} \phi_{j}(y) w_{j}(t), \quad W_{2}(y, t)=\sum_{j=1}^{\infty} \varphi_{j}(y) w_{j}(t)
$$

Due to the independence of the $w_{j}$ and the assumption (2.5), the processes $W_{1}(t)$ and $W_{2}(t)$ are two $H_{0}^{1}(\mathcal{O})$-valued Wiener processes, and

$$
\mathbb{E}\left\|R_{1}(t)\right\|_{L^{2}(\mathcal{O})}^{2} \leq t \max _{y \in \mathcal{O}}\left|\frac{\partial \bar{r}}{\partial t}(r(y, t), t)\right|_{\mathbb{R}^{N}}^{2} \sum_{j=1}^{\infty}\left\|\phi_{j}\right\|_{H_{0}^{1}(\mathcal{O})}^{2} \quad \forall t \geq 0
$$

and

$$
\mathbb{E}\left\|R_{2}(t)\right\|_{L^{2}(\mathcal{O})}^{2} \leq t \max _{y \in \mathcal{O}}\left|\frac{\partial \bar{r}}{\partial t}(r(y, t), t)\right|_{\mathbb{R}^{N}}^{2} \sum_{j=1}^{\infty}\left\|\varphi_{j}\right\|_{H_{0}^{1}(\mathcal{O})}^{2} \quad \forall t \geq 0 .
$$

Therefore, $R_{1}(t)$ and $R_{2}(t)$ are two $\mathcal{F}_{t}$-adapted processes belonging to $L^{\infty}\left(0, T ; L^{2}(\Omega \times \mathcal{O})\right)$ for all $T \geq 0$.

Denote

$$
F(y, t)=U(y, t)-W_{1}(y, t), \quad G(y, t)=V(y, t)-W_{2}(y, t) \quad \text { for } y \in \mathcal{O}, t \geq 0 .
$$


Then equations (2.11) can be transformed into the following equations (2.12):

$$
\left\{\begin{aligned}
& d F= {\left[d_{1} \sum_{j, k=1}^{N} \frac{\partial}{\partial y_{j}}\left(a_{j k}\left(F+W_{1}\right)_{y_{k}}\right)+b \cdot \nabla_{y}\left(F+W_{1}\right)+\left(F+W_{1}\right)-\alpha\left(G+W_{2}\right)\right.} \\
&\left.+\gamma\left(F+W_{1}\right)\left(G+W_{2}\right)-\left(F+W_{1}\right)^{3}+R_{1}\right] d t, \quad \text { in } \mathcal{O} \times[0, \infty), \\
& d G= {\left[d_{2} \sum_{j, k=1}^{N} \frac{\partial}{\partial y_{j}}\left(a_{j k}\left(G+W_{2}\right)_{y_{k}}\right)+c \cdot \nabla_{y}\left(G+W_{2}\right)+\left(F+W_{1}\right)\right.} \\
&\left.\quad-\beta\left(G+W_{2}\right)+R_{2}\right] d t, \quad \text { in } \mathcal{O} \times[0, \infty), \\
& F=0, \quad G=0, \quad \text { on } \partial \mathcal{O} \times[0, \infty), \\
& F(y, 0)=u(r(y, 0))-W_{1}(y, 0), \quad V(y, 0)=v(r(y, 0))-W_{2}(y, 0), \quad y \in \mathcal{O} .
\end{aligned}\right.
$$

In the following, in order to show the existence of strong solution, one is required to impose the conditions on $\phi_{j}$ and $\psi_{j}, j=1,2, \ldots$ by

$$
\sum_{j=1}^{\infty}\left\|\Delta \phi_{j}(y)\right\|_{L^{4}(\mathcal{O})}^{4}<\infty, \quad \sum_{j=1}^{\infty}\left\|\Delta \varphi_{j}(y)\right\|_{L^{2}(\mathcal{O})}^{2}<\infty,
$$

rather than the assumption in (2.5).

\section{Existence of strong solutions of SBS (2.13)}

In this section, we will establish the existence and uniqueness of the strong solution for equation (2.13).

For each $T>0$, consider the auxiliary problem for equation (2.13),

$$
\left\{\begin{aligned}
& d F= {\left[d_{1} \sum_{j, k=1}^{N} \frac{\partial}{\partial y_{j}}\left(a_{j k}\left(F+W_{1}\right)_{y_{k}}\right)+b \cdot \nabla_{y}\left(F+W_{1}\right)+\left(F+W_{1}\right)-\alpha\left(G+W_{2}\right)\right.} \\
&\left.+\gamma\left(F+W_{1}\right)\left(G+W_{2}\right)-\left(F+W_{1}\right)^{3}+R_{1}\right] d t, \quad \text { in } \mathcal{O} \times[0, T], \\
& d G= {\left[d_{2} \sum_{j, k=1}^{N} \frac{\partial}{\partial y_{j}}\left(a_{j k}\left(G+W_{2}\right)_{y_{k}}\right)+c \cdot \nabla_{y}\left(G+W_{2}\right)+\left(F+W_{1}\right)\right.} \\
&\left.\quad-\beta\left(G+W_{2}\right)+R_{2}\right] d t, \quad \text { in } \mathcal{O} \times[0, T], \\
& F=0, \quad G=0, \quad \text { on } \partial \mathcal{O} \times[0, T], \\
& F(y, 0)=u(r(y, 0))-W_{1}(y, 0), \quad V(y, 0)=v(r(y, 0))-W_{2}(y, 0), \quad y \in \mathcal{O} .
\end{aligned}\right.
$$

Definition 3.1 (Strong solution) A $\mathcal{F}_{t}$-adapted process $(F, G)=(F(\omega, y, t), G(\omega, y, t))$ defined in $\Omega \times \mathcal{O} \times[0, T]$ is said to be a strong solution for problem (3.1) if

$$
\begin{aligned}
& F \in L^{2}\left(\Omega, L^{2}\left(\tau, T ; H^{2}(\mathcal{O})\right)\right) \cap L^{2}\left(\Omega, C\left([\tau, T] ; H_{0}^{1}(\mathcal{O})\right)\right), \\
& F^{\prime} \in L^{2}\left(\Omega, L^{2}\left(\tau, T ; L^{2}(\mathcal{O})\right)\right), \\
& G \in L^{2}\left(\Omega, L^{2}\left(\tau, T ; H^{2}(\mathcal{O})\right)\right) \cap L^{2}\left(\Omega, C\left([\tau, T] ; H_{0}^{1}(\mathcal{O})\right)\right), \\
& G^{\prime} \in L^{2}\left(\Omega, L^{2}\left(\tau, T ; L^{2}(\mathcal{O})\right)\right),
\end{aligned}
$$

and the initial data conditions in (3.1) are satisfied almost everywhere in their corresponding domains.

Lemma 3.1 ([6]) For any $-\infty<\tau \leq T<+\infty, a_{j k} \in C^{1}(\overline{\mathcal{O}} \times[\tau, T]), b_{k}, c_{k} \in C^{0}(\overline{\mathcal{O}} \times[\tau, T])$. In particular, $a_{j k}, \frac{\partial a_{j k}}{\partial y_{j}}, b_{k}, c_{k} \in L^{\infty}(\mathcal{O} \times(\tau, T)), j, k=1,2, \ldots, N$. Moreover, there exists a 
$\delta=\delta(v, r, \tau, T)>0$ such that, for any $(y, t) \in \mathcal{O} \times[\tau, T]$,

$$
\sum_{j, k=1}^{N} a_{j k}(y, t) \xi_{j} \xi_{k} \geq \delta|\xi|^{2} \quad \text { for any } \xi \in \mathbb{R}^{N}
$$

Lemma 3.2 ([6]) For any $-\infty<\tau \leq T<+\infty$, there exist two positive constants $\delta_{0}$ and $c_{0}$ which depend on $r, \tau, T$ such that for any $u \in H^{2}(\mathcal{O}) \cap H_{0}^{1}(\mathcal{O})$, the following estimate holds:

$$
\begin{aligned}
\delta_{0} \int_{\mathcal{O}}|\Delta u(y)|^{2} d y \leq & \int_{\mathcal{O}} \sum_{k, j=1}^{N} a_{k j}(y, t) u_{y_{k} y_{j}} \Delta u d y \\
& +c_{0} \int_{\mathcal{O}}|u(y)|^{2} d y \quad \text { for any } t \in[\tau, T] .
\end{aligned}
$$

Define the time-dependent bilinear form by

$$
\begin{aligned}
& B[\alpha, \beta, t]=\int_{\mathcal{O}}-d_{1} \sum_{j, k=1}^{N}\left(a_{j k}(y, t) \alpha_{y_{k}}(y, t)\right) \beta_{y_{j}}(y, t)+\sum_{k=1}^{N} b_{k}(y, t) \nabla_{y} \alpha(y, t) \beta(y, t) d y, \\
& D[\alpha, \beta, t]=\int_{\mathcal{O}}-d_{2} \sum_{j, k=1}^{N}\left(a_{j k}(y, t) \alpha_{y_{k}}(y, t)\right) \beta_{y_{j}}(y, t)+\sum_{k=1}^{N} c_{k}(y, t) \nabla_{y} \alpha(y, t) \beta(y, t) d y,
\end{aligned}
$$

for $\alpha, \beta \in H_{0}^{1}(\mathcal{O})$ and $0 \leq t \leq T$.

We can apply the Galerkin argument(see[16-18]) to prove the existence of solution for SBS. Let $\varpi_{k}=\varpi_{k}(y) \in H^{2}(\mathcal{O}) \cap H_{0}^{1}(\mathcal{O})(k=1,2, \ldots)$ be the eigenfunctions of $-\triangle$ on $H_{0}^{1}(\mathcal{O})$, $0<\lambda_{1}<\lambda_{2}<\cdots<\lambda_{n} \cdots, \lambda_{n} \rightarrow \infty$ as $n \rightarrow \infty$ be the corresponding eigenvalues. Then $\left\{\varpi_{k}\right\}_{k=1}^{\infty}$ is an orthogonal basis of $H_{0}^{1}(\mathcal{O})$ and an orthogonal basis of $L^{2}(\mathcal{O})$.

For each fixed positive integer $m$, denote

$$
F_{m}(t, \omega):=\sum_{k=1}^{m} \zeta_{m}^{k}(t, \omega) \varpi_{k}, \quad G_{m}(t, \omega):=\sum_{k=1}^{m} \eta_{m}^{k}(t, \omega) \varpi_{k} .
$$

Then for $k=1, \ldots, m$ and $\tau \leq t \leq T$,

$$
\begin{aligned}
\left(A_{m}^{1}\right) \quad( & \left.F_{m}^{\prime}(t), \varpi_{k}\right) \\
= & B\left[F_{m}(t)+P_{m} W_{1}, \varpi_{k} ; t\right]+\left(F_{m}(t)+P_{m} W_{1}-\alpha\left(G_{m}(t)+P_{m} W_{2}\right), \varpi_{k}\right) \\
& +\left(\gamma\left(F_{m}(t)+P_{m} W_{1}\right)\left(G_{m}(t)+P_{m} W_{2}\right)-\left(F_{m}(t)+P_{m} W_{1}\right)^{3}+R_{1}, \omega_{k}\right), \\
\left(A_{m}^{2}\right) \quad\left(G_{m}^{\prime}(t), \varpi_{k}\right) & \\
= & D\left[G_{m}(t)+P_{m} W_{2}, \varpi_{k} ; t\right]+\left(F_{m}(t)+P_{m} W_{1}-\beta\left(G_{m}(t)+P_{m} W_{2}\right)+R_{2}, \varpi_{k}\right), \\
F_{m}(0) & =P_{m} F_{0}, \quad G_{m}(0)=P_{m} G_{0},
\end{aligned}
$$

where $F_{0}(y):=u_{0}(r(y, t))-W_{1}, G_{0}(y):=v_{0}(r(y, t))-W_{2} \cdot(\cdot, \cdot)$ is the inner product in $L^{2}(\mathcal{O})$ with associated norm $\|\cdot\|_{L^{2}(\mathcal{O})}, P_{m}$ is the projector from $L^{2}(\mathcal{O})$ to $\operatorname{span}\left\{\varpi_{1}, \varpi_{2}, \ldots, \varpi_{m}\right\}$. It follows from [6] and the assumption (2.5) that $F_{0} \in H_{0}^{1}(\mathcal{O}), G_{0} \in H_{0}^{1}(\mathcal{O})$. 
The assumption (2.14) yields

$$
\begin{aligned}
& P_{m} F_{0} \rightarrow F_{0} \quad \text { in } H_{0}^{1}(\mathcal{O}) \text { as } m \rightarrow \infty, \\
& P_{m} G_{0} \rightarrow G_{0} \quad \text { in } H_{0}^{1}(\mathcal{O}) \text { as } m \rightarrow \infty
\end{aligned}
$$

Noticing that for each integer $m=1,2, \ldots$, there exists a unique local $\mathcal{F}_{t}$-adapted process $\left(F_{m}(\omega), G_{m}(\omega)\right)$ of $(2.7)$ satisfying $\left(A_{m}\right)$ in an interval $\left[0, T_{m}\right]$ with $0 \leq T_{m} \leq T$.

Next, we will show some estimates on the sequences $\left(F_{m}, G_{m}\right), m=1,2, \ldots$

\section{Lemma 3.3 The following estimates hold.}

(1) $\left\{F_{m}\right\}$ is bounded in $C^{0}\left([0, T] ; L^{2}\left(\Omega, L^{2}(\mathcal{O})\right)\right) \cap L^{2}\left(0, T ; L^{2}\left(\Omega, H_{0}^{1}(\mathcal{O})\right)\right) \cap L^{4}([0, T]$; $\left.L^{4}(\mathcal{O} \times \Omega)\right)$

(2) $\left\{G_{m}\right\}$ is bounded in $C^{0}\left([0, T] ; L^{2}\left(\Omega, L^{2}(\mathcal{O})\right)\right) \cap L^{2}\left(0, T ; L^{2}\left(\Omega, H_{0}^{1}(\mathcal{O})\right)\right)$.

Proof Multiplying $\left(A_{m}^{1}\right)$ by $\zeta_{m}^{k}$ and $\left(A_{m}^{2}\right)$ by $\eta_{m}^{k}$, and taking the sum with respect to $k$ from 1 to $m$, we obtain

$$
\begin{aligned}
\frac{1}{2} \frac{d}{d t}\left(\left\|F_{m}\right\|_{L^{2}(\mathcal{O})}^{2}+\left\|G_{m}\right\|_{L^{2}(\mathcal{O})}^{2}\right) \\
=B\left[F_{m}, F_{m} ; t\right]+B\left[P_{m} W_{1}, F_{m} ; t\right]+D\left[G_{m}, G_{m} ; t\right]+D\left[P_{m} W_{2}, G_{m} ; t\right]+\left\|F_{m}(t)\right\|_{L^{2}(\mathcal{O})}^{2} \\
\quad+\left(P_{m} W_{1}, F_{m}\right)-\alpha\left(G_{m}(t)+P_{m} W_{2}, F_{m}(t)\right)+\left(\gamma\left(F_{m}+P_{m} W_{1}\right)\left(G_{m}+P_{m} W_{2}\right), F_{m}\right) \\
\quad-\left(\left(F_{m}+P_{m} W_{1}\right)^{3}, F_{m}\right)+\left(\left(F_{m}+P_{m} W_{1}\right), G_{m}\right)-\left(\beta P_{m} W_{2}, G_{m}\right)-\beta\left\|G_{m}\right\|_{L^{2}(\mathcal{O})}^{2} \\
\quad+\left(R_{1}, F_{m}\right)+\left(R_{2}, G_{m}\right), \quad \forall t \in\left[0, T_{m}\right], \mathbb{P} \text {-a.s. } \omega \in \Omega .
\end{aligned}
$$

Combing Lemma 3.1 with (3.4) and (3.5) guarantees that there exists a positive constant $\delta$, which depends only on $T$ such that $\forall t \in\left[0, T_{m}\right], \mathbb{P}$-a.s. $\omega \in \Omega$,

$$
\begin{aligned}
\frac{1}{2} \frac{d}{d t}( & \left.\left\|F_{m}\right\|_{L^{2}(\mathcal{O})}^{2}+\left\|G_{m}\right\|_{L^{2}(\mathcal{O})}^{2}\right)+\delta\left(d_{1}\left\|F_{m}(t)\right\|_{H_{0}^{1}(\mathcal{O})}^{2}+d_{2}\left\|G_{m}(t)\right\|_{H_{0}^{1}(\mathcal{O})}^{2}\right) \\
\leq & M_{b}\left\|F_{m}\right\|_{L^{2}(\mathcal{O})}^{2}\left\|F_{m}(t)\right\|_{H_{0}^{1}(\mathcal{O})}^{2}+d_{1} M_{a}\left\|P_{m} W_{1}(t)\right\|_{H_{0}^{1}(\mathcal{O})}^{2}\left\|F_{m}\right\|_{H_{0}^{1}(\mathcal{O})}^{2} \\
& +M_{b}\left\|P_{m} W_{1}(t)\right\|_{H_{0}^{1}(\mathcal{O})}^{2}\left\|F_{m}\right\|_{L^{2}(\mathcal{O})}^{2}+M_{c}\left\|G_{m}\right\|_{L^{2}(\mathcal{O})}^{2}\left\|G_{m}(t)\right\|_{H_{0}^{1}(\mathcal{O})}^{2} \\
& +d_{2} M_{a}\left\|P_{m} W_{2}(t)\right\|_{H_{0}^{1}(\mathcal{O})}^{2}\left\|G_{m}\right\|_{H_{0}^{1}(\mathcal{O})}^{2}+M_{c}\left\|P_{m} W_{2}(t)\right\|_{H_{0}^{1}(\mathcal{O})}^{2}\left\|G_{m}\right\|_{L^{2}(\mathcal{O})}^{2} \\
& +\left\|F_{m}(t)\right\|_{L^{2}(\mathcal{O})}^{2}+\left(P_{m} W_{1}, F_{m}\right)-\alpha\left(G_{m}(t)+P_{m} W_{2}, F_{m}(t)\right) \\
& +\left(\gamma\left(F_{m}+P_{m} W_{1}\right)\left(G_{m}+P_{m} W_{2}\right), F_{m}\right)-\left(\left(F_{m}+P_{m} W_{1}\right)^{3}, F_{m}\right) \\
& +\left(\left(F_{m}+P_{m} W_{1}\right), G_{m}\right)-\left(\beta P_{m} W_{2}, G_{m}\right)-\beta\left\|G_{m}\right\|_{L^{2}(\mathcal{O})}^{2}+\left(R_{1}, F_{m}\right)+\left(R_{2}, G_{m}\right)
\end{aligned}
$$

where

$$
M_{a}=N \max _{1 \leq j, k \leq N}\left\|a_{j k}\right\|_{L^{\infty}(\mathcal{O} \times[0, T])}
$$

and

$$
M_{b}=N^{1 / 2} \max _{1 \leq k \leq N}\left\|b_{k}\right\|_{L^{\infty}(\mathcal{O} \times[0, T])}, \quad M_{c}=N^{1 / 2} \max _{1 \leq k \leq N}\left\|c_{k}\right\|_{L^{\infty}(\mathcal{O} \times[0, T])}
$$


Here, we just consider the following term:

$$
\begin{aligned}
\left(\gamma\left(F_{m}+P_{m} W_{1}\right)\left(G_{m}+P_{m} W_{2}\right), F_{m}\right)-\left(\left(F_{m}+P_{m} W_{1}\right)^{3}, F_{m}\right) \\
=\left(\gamma\left(F_{m}+P_{m} W_{1}\right)\left(G_{m}+P_{m} W_{2}\right), F_{m}+P_{m} W_{1}\right)-\left\|F_{m}\right\|_{L^{4}(\mathcal{O})}^{4} \\
\quad-\left(\gamma\left(F_{m}+P_{m} W_{1}\right)\left(G_{m}+P_{m} W_{2}\right), P_{m} W_{1}\right)+\left(\left(F_{m}+P_{m} W_{1}\right)^{3}, P_{m} W_{1}\right) \\
\leq-\frac{1}{8}\left\|F_{m}\right\|_{L^{4}(\mathcal{O})}^{4}+\frac{1}{2}\left\|P_{m} W_{1}\right\|_{L^{4}(\mathcal{O})}^{4}+10 \gamma^{2}\left\|G_{m}\right\|_{L^{2}(\mathcal{O})}^{2}++10 \gamma^{2}\left\|P_{m} W_{2}\right\|_{L^{2}(\mathcal{O})}^{2} .
\end{aligned}
$$

Then it follows from Cauchy's inequality that

$$
\begin{aligned}
& \frac{d}{d t}\left(\left\|F_{m}\right\|_{L^{2}(\mathcal{O})}^{2}+\left\|G_{m}\right\|_{L^{2}(\mathcal{O})}^{2}\right)+\delta\left(d_{1}\left\|F_{m}(t)\right\|_{H_{0}^{1}(\mathcal{O})}^{2}+d_{2}\left\|G_{m}(t)\right\|_{H_{0}^{1}(\mathcal{O})}^{2}\right) \\
& \quad+\frac{1}{4}\left\|F_{m}+P_{m} W_{1}\right\|_{L^{4}(\mathcal{O})}^{4} \leq M_{2}\left\|F_{m}(t)\right\|_{L^{2}(\mathcal{O})}^{2}+M_{3}\left\|G_{m}(t)\right\|_{L^{2}(\mathcal{O})}^{2}+R_{3}(t),
\end{aligned}
$$

where

$$
M_{2}=\frac{2 M_{b}^{2}}{d_{1} \delta}+M_{b}+2 \alpha+5, \quad M_{3}=\frac{2 M_{c}^{2}}{d_{1} \delta}+M_{c}+\alpha+20 \gamma^{2}-\beta+5
$$

and

$$
\begin{aligned}
R_{3}(t)= & \left(\frac{2 d_{1} M_{a}^{2}}{\delta}+M_{b}\right)\left\|P_{m} W_{1}\right\|_{H_{0}^{1}(\mathcal{O})}^{2}+\left(\frac{2 d_{2} M_{a}^{2}}{\delta}+M_{c}\right)\left\|P_{m} W_{2}\right\|_{H_{0}^{1}(\mathcal{O})}^{2} \\
& +2\left\|P_{m} W_{1}\right\|_{L^{2}(\mathcal{O})}^{2}+\left(\alpha+20 \gamma^{2}+\beta\right)\left\|P_{m} W_{2}\right\|_{L^{2}(\mathcal{O})}^{2}+\left\|P_{m} W_{1}\right\|_{L^{4}(\mathcal{O})}^{4} \\
& +\left\|R_{1}\right\|_{L^{2}(\mathcal{O})}^{2}+\left\|R_{2}\right\|_{L^{2}(\mathcal{O})}^{2} .
\end{aligned}
$$

By the fact $\left\|P_{m} W_{1}\right\|_{H_{0}^{1}(\mathcal{O})} \leq\left\|W_{1}\right\|_{H_{0}^{1}(\mathcal{O})},\left\|P_{m} W_{2}\right\|_{H_{0}^{1}(\mathcal{O})} \leq\left\|W_{2}\right\|_{H_{0}^{1}(\mathcal{O})}$, the assumption (2.5) and the BDG inequality, we can find that $\mathbb{E} R_{3}(t)<\infty, \forall t \in\left[0, T_{m}\right]$. Then combining (3.10) with the Gronwall inequality and the fact $\left\|P_{m} U_{0}\right\|_{L^{2}(\mathcal{O})}^{2} \leq\left\|U_{0}\right\|_{L^{2}(\mathcal{O})}^{2},\left\|P_{m} V_{0}\right\|_{L^{2}(\mathcal{O})}^{2} \leq$ $\left\|V_{0}\right\|_{L^{2}(\mathcal{O})}^{2}$, we can find a positive constant $M_{4}$ here such that

$$
\begin{aligned}
& \mathbb{E}\left\|F_{m}(t)\right\|_{L^{2}(\mathcal{O})}^{2}+\mathbb{E}\left\|G_{m}(t)\right\|_{L^{2}(\mathcal{O})}^{2}+\int_{0}^{T} \delta\left(d_{1} \mathbb{E}\left\|F_{m}(s)\right\|_{H_{0}^{1}(\mathcal{O})}^{2}+d_{2} \mathbb{E}\left\|G_{m}(s)\right\|_{H_{0}^{1}(\mathcal{O})}^{2}\right) \\
& \quad+\frac{1}{4} \mathbb{E}\left\|F_{m}(s)\right\|_{L^{4}(\mathcal{O})}^{4} d s \leq M_{4},
\end{aligned}
$$

which implies that Lemma 3.3 holds.

\section{Lemma 3.4 The following estimates hold:}

(3) the sequence $\left\{F_{m}\right\}$ is bounded in $C^{0}\left([0, T] ; L^{2}\left(\Omega, H_{0}^{1}(\mathcal{O})\right)\right) \cap L^{2}\left(0, T ; L^{2}\left(\Omega, H^{2}(\mathcal{O})\right)\right)$,

(4) the sequence $\left\{G_{m}\right\}$ is bounded in $C^{0}\left([0, T] ; L^{2}\left(\Omega, H_{0}^{1}(\mathcal{O})\right)\right) \cap L^{2}\left(0, T ; L^{2}\left(\Omega, H^{2}(\mathcal{O})\right)\right)$.

Proof Multiplying $\left(A_{m}^{2}\right)$ by $\lambda_{k} \eta_{m}^{k}(t, \omega)$ and summing over $k=1,2, \ldots$, and recalling the fact that $-\Delta_{y} G_{m}(t)=\sum_{k=1}^{m} \lambda_{k} \eta_{m}^{k}(t, \omega) \varpi_{k}$ equals 0 on $\partial \mathcal{O}$, we obtain from Lemma 3.2

$$
\begin{aligned}
& \frac{1}{2} \frac{d}{d t}\left\|G_{m}(t)\right\|_{H_{0}^{1}(\mathcal{O})}^{2}+d_{2} \delta_{0}\left\|\Delta G_{m}(t)\right\|_{H_{0}^{1}(\mathcal{O})}^{2} \\
& \quad \leq M_{\bar{c}}\left\|G_{m}(t)\right\|_{H_{0}^{1}(\mathcal{O})}\left\|\Delta G_{m}(t)\right\|_{L^{2}(\mathcal{O})}+d_{2} M_{a}\left\|\Delta P_{m} W_{2}(t)\right\|_{L^{2}(\mathcal{O})}\left\|\Delta G_{m}(t)\right\|_{L^{2}(\mathcal{O})}
\end{aligned}
$$




$$
\begin{aligned}
& +M_{\bar{c}}\left\|P_{m} W_{2}(t)\right\|_{H_{0}^{1}(\mathcal{O})}\left\|\Delta G_{m}(t)\right\|_{L^{2}(\mathcal{O})}+d_{2} C_{0}\left|G_{m}(t)\right|^{2} \\
& -\int_{\mathcal{O}}\left(F_{m}+P_{m} W_{1}-R_{2}\right) \triangle G_{m}+\beta\left(\left(\nabla G_{m}\right)^{2}+\nabla G_{m} \nabla\left(P_{m} W_{2}\right)\right) d y,
\end{aligned}
$$

where $M_{\bar{c}}=N^{1 / 2} \max _{1 \leq k \leq N}\left|\bar{c}_{k}\right|_{L^{\infty}(\mathcal{O} \times(0, T))}$, and $\bar{c}_{k}(y, t):=c_{k}(y, t)+d_{2} \sum_{j=1}^{N} \frac{\partial a_{j k}}{\partial y_{j}}(y, t), k=$ $1,2, \ldots, N$.

By Cauchy's inequality, one derives that

$$
\begin{aligned}
\frac{d}{d t} \| & G_{m}(t)\left\|_{H_{0}^{1}(\mathcal{O})}^{2}+d_{2} \delta_{0}\right\| \Delta G_{m}(t) \|_{L^{2}(\mathcal{O})}^{2} \\
\leq & \left(\frac{4 M_{\bar{C}}^{2}}{d_{2} \delta_{0}}-\beta\right)\left\|G_{m}(t)\right\|_{H_{0}^{1}(\mathcal{O})}^{2}+2 d_{2} c_{0}\left\|G_{m}(t)\right\|_{L^{2}(\mathcal{O})}^{2} \\
& +\frac{4 d_{2} M_{a}^{2}}{\delta_{0}}\left\|\Delta P_{m} W_{2}\right\|_{L^{2}(\mathcal{O})}^{2} \\
& +\left(\frac{4 M_{\bar{C}}^{2}}{d_{2} \delta_{0}}+\beta\right)\left\|P_{m} W_{2}(t)\right\|_{H_{0}^{1}(\mathcal{O})}^{2} \\
& +\frac{3}{d_{2} \delta_{0}}\left(\left\|F_{m}\right\|_{L^{2}(\mathcal{O})}^{2}+\left\|P_{m} W_{1}\right\|_{L^{2}(\mathcal{O})}^{2}+\left\|R_{2}\right\|_{L^{2}(\mathcal{O})}^{2}\right) .
\end{aligned}
$$

Since $P_{m} G_{0}$ is bounded in $H_{0}^{1}(\mathcal{O})$, then (2.14), (3.11), Lemma 3.3 and the Gronwall inequality imply that there exists a positive constant $M_{5}$ that satisfies

$$
\mathbb{E}\left\|G_{m}(t)\right\|_{H_{0}^{1}(\mathcal{O})}^{2}+d_{2} \delta_{0} \int_{0}^{T} \mathbb{E}\left\|\Delta G_{m}(s)\right\|_{L^{2}(\mathcal{O})}^{2} d s \leq M_{5}
$$

Next, we show the second result in Lemma 3.4. Multiplying $\left(A_{m}^{1}\right)$ by $\lambda_{k} \zeta_{m}^{k}$, summing over $k=1,2, \ldots, m$, we get

$$
\begin{aligned}
\frac{1}{2} \frac{d}{d t} & \left\|F_{m}(t)\right\|_{H_{0}^{1}(\mathcal{O})}^{2}+d_{1} \delta_{0}\left\|\Delta F_{m}(t)\right\|_{L^{2}(\mathcal{O})}^{2} \\
\leq & M_{\bar{b}}\left\|F_{m}(t)\right\|_{H_{0}^{1}(\mathcal{O})}\left\|\Delta F_{m}(t)\right\|_{L^{2}(\mathcal{O})}+d_{1} M_{a}\left\|\Delta P_{m} W_{1}(t)\right\|_{L^{2}(\mathcal{O})}\left\|\Delta F_{m}(t)\right\|_{L^{2}(\mathcal{O})} \\
& +M_{\bar{b}}\left\|P_{m} W_{1}(t)\right\|_{H_{0}^{1}(\mathcal{O})}\left\|\Delta F_{m}(t)\right\|_{L^{2}(\mathcal{O})}+d_{1} C_{0}\left|F_{m}(t)\right|^{2}+\left\|F_{m}(t)\right\|_{H_{0}^{1}(\mathcal{O})}^{2} \\
& -\left(P_{m} W_{1}, \Delta F_{m}\right)+\alpha\left(G_{m}(t)+P_{m} W_{2}, \Delta F_{m}\right) \\
& -\left(\gamma\left(F_{m}+P_{m} W_{1}\right)\left(G_{m}+P_{m} W_{2}\right), \triangle F_{m}\right) \\
& -\left(R_{1}, \triangle F_{m}\right)+\left(\left(F_{m}+P_{m} W_{1}\right)^{3}, \Delta F_{m}\right),
\end{aligned}
$$

where $M_{\bar{b}}=N^{1 / 2} \max _{1 \leq k \leq N}\left|\overline{b_{k}}\right|_{L^{\infty}(\mathcal{O} \times(\tau, T))}$, and $\bar{b}_{k}(y, t):=b_{k}(y, t)+d_{1} \sum_{j=1}^{N} \frac{\partial a_{j k}}{\partial y_{j}}(y, t), k=$ $1,2, \ldots, N$. Here, we just consider the last term in (3.12),

$$
\begin{aligned}
& \left(\left(F_{m}+P_{m} W_{1}\right)^{3}, \triangle F_{m}\right) \\
& \quad=\left(\left(F_{m}+P_{m} W_{1}\right)^{3}, \triangle\left(F_{m}+P_{m} W_{1}\right)\right)-\left(\left(F_{m}+P_{m} W_{1}\right)^{3}, \triangle\left(P_{m} W_{1}\right)\right) \\
& \quad=-3 \int_{\mathcal{O}}\left(F_{m}+P_{m} W_{1}\right)^{2}\left(\nabla\left(F_{m}+P_{m} W_{1}\right)\right)^{2} d y-\left(\left(F_{m}+P_{m} W_{1}\right)^{3}, \triangle\left(P_{m} W_{1}\right)\right)
\end{aligned}
$$




$$
\begin{aligned}
& \leq-\left(\left(F_{m}+P_{m} W_{1}\right)^{3}, \triangle\left(P_{m} W_{1}\right)\right) \\
& \leq \frac{3}{4}\left\|F_{m}+P_{m} W_{1}\right\|_{L^{4}(\mathcal{O})}^{4}+\frac{1}{4}\left\|\Delta\left(P_{m} W_{1}\right)\right\|_{L^{4}(\mathcal{O})}^{4} .
\end{aligned}
$$

The Cauchy inequality implies that

$$
\begin{aligned}
& \frac{d}{d t}\left\|F_{m}\right\|_{H^{1}(\mathcal{O})}^{2}+d_{1} \delta_{0}\left\|\Delta F_{m}\right\|_{L^{2}(\mathcal{O})}^{2} \\
& \leq\left(\frac{4 M_{\bar{b}}^{2}}{d_{1} \delta_{0}}+\alpha+3\right)\left\|F_{m}\right\|_{H_{0}^{1}(\mathcal{O})}^{2}+2 d_{1} C_{0}\left\|F_{m}\right\|_{L^{2}(\mathcal{O})}^{2}+\frac{4 d_{1} M_{a}^{2}}{\delta_{0}}\left\|\Delta P_{m} W_{1}\right\|_{L^{2}(\mathcal{O})}^{2} \\
& \quad+\left(\frac{4 M_{\bar{b}}^{2}}{d_{1} \delta_{0}}+1\right)\left\|P_{m} W_{1}\right\|_{H_{0}^{1}(\mathcal{O})}^{2}+\alpha\left\|G_{m}+P_{m} W_{2}\right\|_{H_{0}^{1}(\mathcal{O})}^{2} \\
& \quad+\left(\frac{2 \gamma^{2}}{d_{1} \delta_{0}}+\frac{3}{2}\right)\left\|F_{m}+P_{m} W_{1}\right\|_{L^{4}(\mathcal{O})}^{4} \\
& \quad+\frac{2 \gamma^{2}}{d_{1} \delta_{0}}\left\|G_{m}+P_{m} W_{2}\right\|_{L^{4}(\mathcal{O})}^{4}+\frac{1}{2}\left\|\triangle P_{m} W_{1}\right\|_{L^{4}(\mathcal{O})}^{4} .
\end{aligned}
$$

Then for $N \leq 3$, the assumptions (2.5) and (2.14) imply that the second result of Lemma 3.4 holds.

Lemma 3.5 The sequences $\left\{F_{m}^{\prime}\right\},\left\{G_{m}^{\prime}\right\}$ are bounded in $L^{2}\left(0, T ; L^{2}\left(\Omega, L^{2}(\mathcal{O})\right)\right)$.

Proof Multiplying $\left(A_{m}^{1}\right)$ by $\zeta_{m}^{k \prime}$, summing over $k=1,2, \ldots, m$, and combining with $a_{k, j}=a_{j, k}$, we have

$$
\begin{aligned}
\left\|F_{m}^{\prime}(t)\right\|_{L^{2}(\mathcal{O})}^{2}+\frac{d_{1}}{2} \frac{d}{d t} \int_{\mathcal{O}} \sum_{k, j=1}^{N} a_{k, j}(y, t) \frac{\partial F_{m}}{\partial y_{j}}(y, t) \frac{\partial F_{m}}{\partial y_{k}}(y, t) d y \\
\quad-\frac{d_{1}}{2} \frac{d}{d t} \int_{\mathcal{O}} \sum_{k, j=1}^{N} \frac{\partial a_{k, j}(y, t)}{\partial t} \frac{\partial F_{m}}{\partial y_{j}}(y, t) \frac{\partial F_{m}}{\partial y_{k}}(y, t) d y \\
\leq \frac{7}{8}\left|F_{m}^{\prime}\right|_{L^{2}(\mathcal{O})}^{2}+2 M_{a}^{2} d_{1}^{2}\left\|P_{m} W_{1}\right\|_{H_{0}^{1}(\mathcal{O})}^{2}+2 M_{b}^{2}\left\|F_{m}+P_{m} W_{1}\right\|_{H_{0}^{1}(\mathcal{O})}^{2} \\
\quad+2\left\|F_{m}+P_{m} W_{1}\right\|_{L^{2}(\mathcal{O})}^{2}+2 \alpha^{2}\left\|G_{m}+P_{m} W_{2}\right\|_{L^{2}(\mathcal{O})}^{2} \\
+\gamma^{2}\left(\left\|F_{m}+P_{m} W_{1}\right\|_{L^{4}(\mathcal{O})}^{4}+\left\|G_{m}+P_{m} W_{2}\right\|_{L^{4}(\mathcal{O})}^{4}\right) \\
+2\left\|F_{m}+P_{m} W_{1}\right\|_{L^{6}(\mathcal{O})}^{6}+2\left\|R_{1}\right\|_{L^{2}(\mathcal{O})}^{2} .
\end{aligned}
$$

Similarly,

$$
\begin{aligned}
& \left\|F_{m}^{\prime}(t)\right\|_{L^{2}(\mathcal{O})}^{2}+\frac{d_{2}}{2} \frac{d}{d t} \int_{\mathcal{O}} \sum_{k, j=1}^{N} a_{k, j}(y, t) \frac{\partial G_{m}}{\partial y_{j}}(y, t) \frac{\partial G_{m}}{\partial y_{k}}(y, t) d y \\
& \quad-\frac{d_{2}}{2} \frac{d}{d t} \int_{\mathcal{O}} \sum_{k, j=1}^{N} \frac{\partial a_{k, j}(y, t)}{\partial t} \frac{\partial G_{m}}{\partial y_{j}}(y, t) \frac{G \varphi_{m}}{\partial y_{k}}(y, t) d y \\
& \leq \frac{5}{8}\left|G_{m}^{\prime}\right|_{L^{2}(\mathcal{O})}^{2}+2 M_{a}^{2} d_{2}^{2}\left\|P_{m} W_{2}\right\|_{H_{0}^{1}(\mathcal{O})}^{2}+2 M_{c}^{2}\left\|G_{m}+P_{m} W_{2}\right\|_{H_{0}^{1}(\mathcal{O})}^{2} \\
& \quad+2\left\|F_{m}+P_{m} W_{1}\right\|_{L^{2}(\mathcal{O})}^{2}+2 \beta^{2}\left\|G_{m}+P_{m} W_{2}\right\|_{L^{2}(\mathcal{O})}^{2}+2\left\|R_{2}\right\|_{L^{2}(\mathcal{O})}^{2} .
\end{aligned}
$$


Noticing the fact that $a_{k, j} \in C^{1}(\overline{\mathcal{O}} \times[\tau, T])(k=1,2, \ldots, N), P_{m} F_{0}, P_{m} G_{0}$ are bounded in $H_{0}^{1}(\mathcal{O}), N \leq 3$, we deduce that Lemma 3.5 holds.

Theorem 3.1 Assume that $r$ and $\bar{r}$ satisfy the assumptions (2.1), (2.2), (2.3), and

$$
\partial \mathcal{O} \text { is } C^{m} \quad \text { where } m \geq 2 \text {. }
$$

Then for any $\left(u_{0}, v_{0}\right) \in H_{0}^{1}\left(\mathcal{O}_{0}\right) \times H_{0}^{1}\left(\mathcal{O}_{0}\right),\left\{\phi_{j}(y)\right\}_{j=1,2, \ldots},\left\{\varphi_{j}(y)\right\}_{j=1,2, \ldots}$ satisfy the assumption (2.14), and for any $0 \leq T<+\infty$, there exists a unique strong solution $(F, G)$ of $(3.1)$. Moreover, $(F, G)$ satisfies the equality of energy, for $\mathbb{P}$-a.s. $\omega \in \Omega$,

$$
\begin{aligned}
\frac{1}{2} \frac{d}{d t} & \|F\|_{L^{2}(\mathcal{O})}^{2}+\int_{0}^{T} \int_{\mathcal{O}} d_{1} \sum_{j, k=1}^{N}\left(a_{j k}\left(F+W_{1}\right)_{y k}\right) F_{y j}+\left(F+W_{1}\right) b \cdot \nabla_{y} F d y d t \\
= & \int_{0}^{T} \int_{\mathcal{O}}\left[\left(F+W_{1}\right)-\alpha\left(G+W_{2}\right)+\gamma\left(F+W_{1}\right)\left(G+W_{2}\right)\right. \\
& \left.-\left(F+W_{1}\right)^{3}+R_{1}\right] F d y d t
\end{aligned}
$$

and

$$
\begin{aligned}
& \frac{1}{2} \frac{d}{d t}\|G\|_{L^{2}(\mathcal{O})}^{2}+\int_{0}^{T} \int_{\mathcal{O}} d_{2} \sum_{j, k=1}^{N}\left(a_{j k}\left(G+W_{2}\right)_{y k}\right) G_{y j}+\left(G+W_{2}\right) c \cdot \nabla_{y} G d y d t \\
& \quad=\int_{0}^{T} \int_{\mathcal{O}}\left[\left(F+W_{1}\right)-\beta\left(G+W_{2}\right)+R_{2}\right] G d y d t, \quad \forall t \in[0, T] ;
\end{aligned}
$$

and the following estimates, for $\mathbb{P}$-a.s. $\omega \in \Omega$ :

$$
\begin{aligned}
& \|F(t)\|_{L^{2}(\mathcal{O})}^{2}+\|G(t)\|_{L^{2}(\mathcal{O})}^{2} \leq e^{M t}\left(\left\|F_{0}\right\|_{L^{2}(\mathcal{O})}^{2}+\left\|G_{0}\right\|_{L^{2}(\mathcal{O})}^{2}\right)+\int_{0}^{t} e^{M t} R d s \\
& \delta \int_{0}^{t}\left(d_{1}\|F\|_{H_{0}^{1}(\mathcal{O})}^{2}+d_{2}\|G\|_{H_{0}^{1}(\mathcal{O})}^{2}\right) d s \\
& \quad \leq e^{M t}\left(\left\|F_{0}\right\|_{L^{2}(\mathcal{O})}^{2}+\left\|G_{0}\right\|_{L^{2}(\mathcal{O})}^{2}\right)+\int_{0}^{t} e^{M t} R d s
\end{aligned}
$$

where $M$ is a constant and $R$ is a fixed random function which satisfies, for $\mathbb{P}$-a.s. $\omega \in \Omega$, $R(t) \in L^{1}(0, T)$.

Proof We first prove the uniqueness of the solution. Let $\left(u_{i 0}, v_{i 0}\right) \in H_{0}^{1}\left(\mathcal{O}_{0}\right) \times H_{0}^{1}\left(\mathcal{O}_{0}\right)$ and $\left(F_{i}(t), G_{i}(t)\right), i=1,2$ be the corresponding strong solutions, then we derive

$$
\begin{aligned}
\frac{\partial\left(U_{1}-U_{2}\right)}{\partial t}= & d_{1} \sum_{j, k=1}^{N} \frac{\partial}{\partial y_{j}}\left(a_{j k}\left(U_{1}-U_{2}\right)_{y_{k}}\right)+b \cdot \nabla_{y}\left(U_{1}-U_{2}\right)+\left(U_{1}-U_{2}\right) \\
& -\alpha\left(V_{1}-V_{2}\right)+\gamma\left(U_{1}-U_{2}\right)\left(V_{1}-V_{2}\right)-\left(\left(U_{1}\right)^{3}-\left(U_{2}\right)^{3}\right) \\
\frac{\partial\left(V_{1}-V_{2}\right)}{\partial t}= & d_{2} \sum_{j, k=1}^{N} \frac{\partial}{\partial y_{j}}\left(a_{j k}\left(V_{1}-V_{2}\right)_{y_{k}}\right)+c \cdot \nabla_{y}\left(V_{1}-V_{2}\right)+\left(U_{1}-U_{2}\right) \\
& -\beta\left(V_{1}-V_{2}\right) .
\end{aligned}
$$


Taking the inner product of (3.18) with $\left(U_{1}-U_{2}\right)$ and (3.19) with $\alpha\left(V_{1}-V_{2}\right)$ in $L^{2}\left(\mathcal{O}_{t}\right)$, we obtain, $\mathbb{P}$-a.s. $\omega \in \Omega$,

$$
\begin{aligned}
\frac{d}{d t}( & \left.\left\|\left(U_{1}-U_{2}\right)\right\|_{L^{2}(\mathcal{O})}^{2}+\alpha\left\|\left(V_{1}-V_{2}\right)\right\|_{L^{2}(\mathcal{O})}^{2}\right) \\
& +d_{1} \delta\left\|\nabla_{y}\left(U_{1}-U_{2}\right)\right\|_{L^{2}(\mathcal{O})}^{2}+\alpha d_{2} \delta\left\|\nabla_{y}\left(V_{1}-V_{2}\right)\right\|_{L^{2}(\mathcal{O})}^{2} \\
\leq & \left(2+\frac{M_{b}^{2}}{d_{1} \delta}\right)\left\|U_{1}-U_{2}\right\|_{L^{2}(\mathcal{O})}^{2}+\alpha\left(\frac{M_{c}^{2}}{d_{2} \delta}-2 \beta\right)\left\|V_{1}-V_{2}\right\|_{t}^{2} \\
& +2 \int_{\mathcal{O}} \gamma\left(U_{1}\left(V_{1}-V_{2}\right)+V_{2}\left(U_{1}-U_{2}\right)\right) d y
\end{aligned}
$$

where $M_{b}, M_{c}$ are defined by (3.9).

Thanks to the Hölder inequality

$$
\begin{aligned}
\int_{\mathcal{O}}( & \left.U_{1}\left(V_{1}-V_{2}\right)+V_{2}\left(U_{1}-U_{2}\right)\right) d y \\
\leq & \left\|U_{1}\right\|_{L^{4}(\mathcal{O})}\left\|V_{1}-V_{2}\right\|_{L^{4}(\mathcal{O})}\left\|U_{1}-U_{2}\right\|_{L^{2}(\mathcal{O})} \\
& +\left\|V_{2}\right\|_{L^{4}(\mathcal{O})}\left\|U_{1}-U_{2}\right\|_{L^{4}(\mathcal{O})}\left\|V_{1}-V_{2}\right\|_{L^{2}(\mathcal{O})} .
\end{aligned}
$$

Since $\left(F_{1}, G_{1}\right)$ and $\left(F_{2}, G_{2}\right)$ are strong solutions of (3.1), $U_{1}, V_{2} \in H_{0}^{1}(\mathcal{O}), \forall t \in[\tau, T]$, and there exists a constant $M$ such that $\left\|U_{1}\right\|_{H_{0}^{1}(\mathcal{O})} \leq M$ and $\left\|V_{2}\right\|_{H_{0}^{1}(\mathcal{O})} \leq M$. Applying the Sobolev embedding theorem, Cauchy's inequality, and (3.21), we have

$$
\begin{aligned}
\int_{\mathcal{O}} & \gamma\left(U_{1}\left(V_{1}-V_{2}\right)+V_{2}\left(U_{1}-U_{2}\right)\right) d y \\
\leq & \frac{d_{1} \delta}{4}\left\|\left(U_{1}-U_{2}\right)\right\|_{H_{0}^{1}(\mathcal{O})}^{2}+\frac{\alpha d_{2} \delta}{4}\left\|\left(V_{1}-V_{2}\right)\right\|_{H_{0}^{1}(\mathcal{O})}^{2} \\
& +\tilde{M}\left(\left\|U_{1}-U_{2}\right\|_{L^{2}(\mathcal{O})}^{2}+\left\|V_{1}-V_{2}\right\|_{L^{2}(\mathcal{O})}^{2}\right),
\end{aligned}
$$

where $\tilde{M}$ is a constant dependent on $d_{1}, d_{2}, M, \alpha, \gamma, \delta$, and the Sobolev embedding constant.

Combining (3.22) with (3.20) yields

$$
\begin{aligned}
& \frac{d}{d t}\left(\left\|\left(U_{1}-U_{2}\right)\right\|_{L^{2}(\mathcal{O})}^{2}+\alpha\left\|\left(V_{1}-V_{2}\right)\right\|_{L^{2}(\mathcal{O})}^{2}\right)+\frac{d_{1} \delta}{2}\left\|\left(U_{1}-U_{2}\right)\right\|_{H_{0}^{1}(\mathcal{O})}^{2} \\
& \quad+\frac{\alpha d_{2} \delta}{2}\left\|\left(V_{1}-V_{2}\right)\right\|_{\left.H_{0}^{1}(\mathcal{O})\right)}^{2} \\
& \quad \leq M_{1}\left(\left\|\left(U_{1}-U_{2}\right)\right\|_{L^{2}(\mathcal{O})}^{2}+\left\|\left(V_{1}-V_{2}\right)\right\|_{L^{2}(\mathcal{O})}^{2}\right),
\end{aligned}
$$

where $M_{1}=\max \left\{1, \frac{1}{\alpha}\right\} * \max \left\{2 \tilde{M}+\left(2+\frac{M_{b}^{2}}{d_{1} \delta}\right), 2 \tilde{M}+\alpha\left(\frac{M_{c}^{2}}{d_{2} \delta}-2 \beta\right)\right\}$.

Due to the Gronwall lemma and the fact $u_{10}(x)-u_{20}(x)=v_{10}(x)-v_{20}(x)=0, F_{1}-F_{2}=U_{1}-$ $U_{2}, G_{1}-G_{2}=V_{1}-V_{2}$, we obtain the uniqueness of the strong solution for (3.1) immediately. Taking the inner product of (3.1) with $(U, V)$, we can obtain the energy equality (3.14) and (3.15) immediately.

Based on the estimates in Lemma 3.3, Lemma 3.4, and Lemma 3.5 on $F_{m}$ and $G_{m}$, there exist a subsequence of $\left\{F_{m}(\omega)\right\}$ and a subsequence of $\left\{G_{m}(\omega)\right\}$ converging weakly in 
$L^{2}\left((0, T] \times ; H^{2}(\mathcal{O})\right)$, weakly star in $L^{\infty}\left(0, T ; H_{0}^{1}(\mathcal{O})\right)$, and strongly in $L^{2}\left((0, T] ; H_{0}^{1}(\mathcal{O})\right)$, for $\mathbb{P}$-a.s. $\omega \in \Omega$. Moreover, the extremities $F(\omega), G(\omega)$ are $\mathcal{F}$-adapted processes and satisfy

$$
\begin{aligned}
& F \in L^{2}\left(\Omega, L^{2}\left(\tau, T ; H^{2}(\mathcal{O})\right)\right) \cap L^{2}\left(\Omega, C\left([\tau, T] ; H_{0}^{1}(\mathcal{O})\right)\right), \\
& F^{\prime} \in L^{2}\left(\Omega, L^{2}\left(\tau, T ; L^{2}(\mathcal{O})\right)\right),
\end{aligned}
$$

and

$$
\begin{aligned}
& G \in L^{2}\left(\Omega, L^{2}\left(\tau, T ; H^{2}(\mathcal{O})\right)\right) \cap L^{2}\left(\Omega, C\left([\tau, T] ; H_{0}^{1}(\mathcal{O})\right)\right), \\
& G^{\prime} \in L^{2}\left(\Omega, L^{2}\left(\tau, T ; L^{2}(\mathcal{O})\right)\right) .
\end{aligned}
$$

Thus, $\left\{\left(F_{m}, G_{m}\right)\right\}$ converges to $(F, G)$ in the sense of mean-square.

Therefore, it follows that, for $\mathbb{P}$-a.s. $\omega \in \Omega$,

$$
\begin{aligned}
& \frac{d}{d t}\left(\|F\|_{L^{2}(\mathcal{O})}^{2}+\|G\|_{L^{2}(\mathcal{O})}^{2}\right)+\delta\left(d_{1}\|F(t)\|_{H_{0}^{1}(\mathcal{O})}^{2}+d_{2}\|G(t)\|_{H_{0}^{1}(\mathcal{O})}^{2}\right)+\frac{7}{8}\|F\|_{L^{4}(\mathcal{O})}^{4} \\
& \quad \leq M_{2}\|F(t)\|_{L^{2}(\mathcal{O})}^{2}+M_{3}\|G(t)\|_{L^{2}(\mathcal{O})}^{2}+R(t)
\end{aligned}
$$

where

$$
\begin{aligned}
R(t)= & \left(2 d_{1} M_{a}^{2}+M_{b}\right)\left\|W_{1}\right\|_{H_{0}^{1}(\mathcal{O})}^{2}+\left(2 d_{2} M_{a}^{2}+M_{c}\right)\left\|W_{2}\right\|_{H_{0}^{1}(\mathcal{O})}^{2}+2\left\|W_{1}\right\|_{L^{2}(\mathcal{O})}^{2} \\
& +\left(\alpha+4 \gamma^{2}+\beta\right)\left\|W_{2}\right\|_{L^{2}(\mathcal{O})}^{2}+\left(\frac{\gamma}{2}+2 \gamma^{4}+348\right)\left\|W_{1}\right\|_{L^{4}(\mathcal{O})}^{4} \\
& +\frac{\gamma}{2}\left\|W_{2}\right\|_{L^{4}(\mathcal{O})}^{4}+\left\|R_{1}\right\|_{L^{2}(\mathcal{O})}^{2}+\left\|R_{2}\right\|_{L^{2}(\mathcal{O})}^{2} .
\end{aligned}
$$

Denote $M=\max \left\{M_{2}, M_{3}\right\}$; the Gronwall inequality implies Theorem 3.1 holds.

\section{Existence of the weak solution}

In this section, we will show the existence of the weak solution for SBS.

Denote

$$
\begin{aligned}
\mathcal{U}_{\tau, T}:= & \left\{\vartheta \in L^{2}\left(0, T ; H_{0}^{1}(\mathcal{O})\right) \cap L^{4}\left(0, T ; L^{4}(\mathcal{O})\right): \vartheta^{\prime} \in L^{2}\left(0, T ; L^{2}(\mathcal{O})\right),\right. \\
& \vartheta(0)=\vartheta(T)=0\}
\end{aligned}
$$

Definition 4.1 For any given initial data $\left(u_{0}, v_{0}\right) \in\left(L^{2}\left(\mathcal{O}_{0}\right)\right)^{2}, 0 \leq T<+\infty$, a function $(F, G)$ is called a weak solution of (3.1) if the following conditions hold. $\mathbb{P}$-a.s. $\omega \in \Omega$,

(1) $F \in C\left([0, T] ; L^{2}(\mathcal{O})\right) \cap L^{2}\left([0, T] ; H_{0}^{1}(\mathcal{O})\right) \cap L^{4}\left(0, T ; L^{4}(\mathcal{O})\right)$, $G \in C\left([0, T] ; L^{2}(\mathcal{O})\right) \cap L^{2}\left([0, T] ; H_{0}^{1}(\mathcal{O})\right)$ with $(F(0), G(0))=\left(u_{0}(r(y, 0))+W_{1}(0), v_{0}(r(y, 0))+W_{2}(0)\right)$.

(2) There exists a sequence of regular data $\left(F_{0, m}, G_{0, m}\right) \in H_{0}^{1}(\mathcal{O}) \times H_{0}^{1}(\mathcal{O}), m=1,2, \ldots$, such that $\left(F_{0, m}, G_{0, m}\right) \rightarrow\left(F_{0}, G_{0}\right)$ in $L^{2}(\mathcal{O}) \times L^{2}(\mathcal{O})$ and $\left(F_{m}, G_{m}\right) \rightarrow(F, G)$ in $C\left([0, T] ; L^{2}(\mathcal{O} \times \Omega)\right) \times C\left([0, T] ; L^{2}(\mathcal{O} \times \Omega)\right)$. 
(3) It follows that, for all $\vartheta \in \mathcal{U}_{0, T}$,

$$
\begin{aligned}
\int_{0}^{T} & \int_{\mathcal{O}}-F \vartheta^{\prime}+d_{1} \sum_{j, k=1}^{N}\left(a_{j k}\left(F+W_{1}\right)_{y k}\right) \vartheta_{y j}+\left(F+W_{1}\right) b \cdot \nabla_{y} \vartheta d y d t \\
= & \int_{0}^{T} \int_{\mathcal{O}}\left[\left(F+W_{1}\right)-\alpha\left(G+W_{2}\right)+\gamma\left(F+W_{1}\right)\left(G+W_{2}\right)\right. \\
& \left.-\left(F+W_{1}\right)^{3}+R_{1}\right] \vartheta d y d t
\end{aligned}
$$

and

$$
\begin{gathered}
\int_{0}^{T} \int_{\mathcal{O}}-G \vartheta^{\prime}+d_{2} \sum_{j, k=1}^{N}\left(a_{j k}\left(G+W_{2}\right)_{y k}\right) \vartheta_{y j}+\left(G+W_{2}\right) c \cdot \nabla_{y} \vartheta d y d t \\
=\int_{0}^{T} \int_{\mathcal{O}}\left[\left(F+W_{1}\right)-\beta\left(G+W_{2}\right)+R_{2}\right] \vartheta d y d t .
\end{gathered}
$$

It is easy to find that every strong solution is a weak solution of (3.1) from the definition.

Theorem 4.1 Let the function $r$ and $\bar{r}$ satisfy assumptions (2.1)-(2.3). Assume that $\partial \mathcal{O}$ is $C^{m} m \geq 2$. Then for any $\left(F_{0}, G_{0}\right) \in L^{2}(\mathcal{O}) \times L^{2}(\mathcal{O})$ and $0 \leq T<+\infty$, there exists a unique weak solution $(F, G)$ of (3.1). Moreover, $(F, G)$ satisfies the equality of energy, for $\mathbb{P}$-a.s. $\omega \in \Omega$,

$$
\begin{aligned}
& \frac{1}{2} \frac{d}{d}\|F\|_{L^{2}(\mathcal{O})}^{2}+\int_{0}^{T} \int_{\mathcal{O}} d_{1} \sum_{j, k=1}^{N}\left(a_{j k}\left(F+W_{1}\right)_{y k}\right) F_{y j}+\left(F+W_{1}\right) b \cdot \nabla_{y} F d y d t \\
& =\int_{0}^{T} \int_{\mathcal{O}}\left[\left(F+W_{1}\right)-\alpha\left(G+W_{2}\right)+\gamma\left(F+W_{1}\right)\left(G+W_{2}\right)\right. \\
& \left.\quad-\left(F+W_{1}\right)^{3}+R_{1}\right] F d y d t, \quad \forall t \in[0, T] ; \\
& \frac{1}{2} \frac{d}{d}\|G\|_{L^{2}(\mathcal{O})}^{2}+\int_{0}^{T} \int_{\mathcal{O}} d_{2} \sum_{j, k=1}^{N}\left(a_{j k}\left(G+W_{2}\right)_{y k}\right) G_{y j}+\left(G+W_{2}\right) c \cdot \nabla_{y} G d y d t \\
& =\int_{0}^{T} \int_{\mathcal{O}}\left[\left(F+W_{1}\right)-\beta\left(G+W_{2}\right)+R_{2}\right] G d y d t, \quad \forall t \in[0, T],
\end{aligned}
$$

and the following estimates, for $\mathbb{P}$-a.s. $\omega \in \Omega$ :

$$
\begin{aligned}
& \|F(t)\|_{L^{2}(\mathcal{O})}^{2}+\|G(t)\|_{L^{2}(\mathcal{O})}^{2} \leq e^{M t}\left(\left\|F_{0}\right\|_{L^{2}(\mathcal{O})}^{2}+\left\|G_{0}\right\|_{L^{2}(\mathcal{O})}^{2}\right)+\int_{0}^{t} e^{M t} R d s, \\
& \delta \int_{0}^{t}\left(d_{1}\|F\|_{H_{0}^{1}(\mathcal{O})}^{2}+d_{2}\|G\|_{H_{0}^{1}(\mathcal{O})}^{2}\right) d s \leq e^{M t}\left(\left\|F_{0}\right\|_{L^{2}(\mathcal{O})}^{2}+\left\|G_{0}\right\|_{L^{2}(\mathcal{O})}^{2}\right)+\int_{0}^{t} e^{M t} R d s,
\end{aligned}
$$

where $M$ and $R$ are defined in the proof of Theorem 3.1.

Proof We first of all show the uniqueness of weak solutions for (3.1). Let $\left(F_{1}, G_{1}\right)$ and $\left(F_{2}, G_{2}\right)$ be weak solutions for (3.1) with the initial value $\left(u_{0,1}, v_{0,1}\right)$ and $\left(u_{0,2}, v_{0,2}\right)$, respec- 
tively, then

$$
\begin{aligned}
& \left(B_{1}\right) \quad \frac{\partial\left(U_{1}-U_{2}\right)}{\partial t}=d_{1} \sum_{j, k=1}^{N} \frac{\partial}{\partial y_{j}}\left(a_{j k}\left(U_{1}-U_{2}\right)_{y_{k}}\right)+b \cdot \nabla_{y}\left(U_{1}-U_{2}\right)+\left(U_{1}-U_{2}\right) \\
& -\alpha\left(V_{1}-V_{2}\right)+\gamma\left(U_{1}-U_{2}\right)\left(V_{1}-V_{2}\right)-\left(\left(U_{1}\right)^{3}-\left(U_{2}\right)^{3}\right), \\
& \left(B_{2}\right) \quad \frac{\partial\left(V_{1}-V_{2}\right)}{\partial t}=d_{2} \sum_{j, k=1}^{N} \frac{\partial}{\partial y_{j}}\left(a_{j k}\left(V_{1}-V_{2}\right)_{y_{k}}\right)+c \cdot \nabla_{y}\left(V_{1}-V_{2}\right)+\left(U_{1}-U_{2}\right) \\
& -\beta\left(V_{1}-V_{2}\right) \text {. }
\end{aligned}
$$

Taking the inner product of $\left(B_{2}\right)$ with $V_{1}-V_{2}$ in $L^{2}(\mathcal{O})$ and using Lemma 3.1 and Cauchy's inequality, we obtain

$$
\begin{aligned}
& \frac{d}{d t}\left\|V_{1}-V_{2}\right\|_{L^{2}(\mathcal{O})}^{2}+d_{2} \delta\left\|\left(V_{1}-V_{2}\right)\right\|_{H_{0}^{1}(\mathcal{O})}^{2} \\
& \quad \leq\left(\frac{M_{c}^{2}}{d_{2} \delta}-2 \beta+1\right)\left\|V_{1}-V_{2}\right\|_{L^{2}(\mathcal{O})}^{2}+\left\|U_{1}-U_{2}\right\|_{L^{2}(\mathcal{O})}^{2},
\end{aligned}
$$

where $M_{c}$ is defined in the proof of Theorem 3.1.

Taking the inner product of $\left(B_{1}\right)$ with $U_{1}-U_{2}$ in $L^{2}(\mathcal{O})$ and using Lemma 3.1 and Cauchy's inequality again, we can get

$$
\begin{aligned}
\frac{d}{d t} & \left\|U_{1}-U_{2}\right\|_{L^{2}(\mathcal{O})}^{2}+d_{1} \delta\left\|\left(U_{1}-U_{2}\right)\right\|_{H_{0}^{1}(\mathcal{O})}^{2} \\
\leq & \left(\frac{M_{b}^{2}}{d_{1} \delta}+2\right)\left\|U_{1}-U_{2}\right\|_{L^{2}(\mathcal{O})}^{2} \\
& +2 \int_{\mathcal{O}}\left(-\alpha\left(V_{1}-V_{2}\right)+\gamma\left(U_{1} V_{1}-U_{2} V_{2}\right)-\left(U_{1}^{3}-U_{2}^{3}\right)\right)\left(U_{1}-U_{2}\right) d y \\
\leq & \left(\frac{M_{b}^{2}}{d_{1} \delta}+\alpha+2\right)\left\|U_{1}-U_{2}\right\|_{L^{2}(\mathcal{O})}^{2}+\alpha\left\|V_{1}-V_{2}\right\|_{L^{2}(\mathcal{O})}^{2} \\
& +2 \int_{\mathcal{O}} \gamma\left(U_{1} V_{1}-U_{2} V_{2}\right)\left(U_{1}-U_{2}\right) d y .
\end{aligned}
$$

Notice that $U_{1}, U_{2} \in C\left(0, T ; L^{2}(\mathcal{O})\right)$, then there exists a constant $M_{u}$ such that $\left|U_{1}\right|_{t}^{2}+$ $\left|U_{2}\right|_{t}^{2} \leq M_{u}, \forall t \in(0, T)$, and

$$
\begin{aligned}
& \int_{\mathcal{O}}\left(\gamma\left(U_{1} V_{1}-U_{2} V_{2}\right)\right)\left(U_{1}-U_{2}\right) d y \\
& \quad=\int_{\mathcal{O}} \gamma U_{1}\left(V_{1}-V_{2}\right)\left(U_{1}-U_{2}\right)+V_{2}\left(U_{1}-U_{2}\right)^{2} d y \\
& \quad \leq \gamma M_{u}\left\|V_{1}-V_{2}\right\|_{L^{4}(\mathcal{O})}\left\|U_{1}-U_{2}\right\|_{L^{4}(\mathcal{O})}+\gamma\left\|V_{2}\right\|_{L^{4}(\mathcal{O})}\left\|U_{1}-U_{2}\right\|_{L^{2}(\mathcal{O})}^{2}\left\|U_{1}-U_{2}\right\|_{L^{4}(\mathcal{O})} .
\end{aligned}
$$

Hence, there exists a constant $C_{N}$ such that

$$
\begin{aligned}
& \int_{\mathcal{O}}\left(\gamma\left(U_{1} V_{1}-U_{2} V_{2}\right)\right)\left(U_{1}-U_{2}\right) d y \\
& \quad \leq \gamma M_{u} C_{N}^{2}\left\|V_{1}-V_{2}\right\|_{H_{0}^{1}(\mathcal{O})}\left\|U_{1}-U_{2}\right\|_{H_{0}^{1}(\mathcal{O})}
\end{aligned}
$$




$$
\begin{aligned}
& +\gamma C_{N}^{2}\left\|V_{2}\right\|_{H_{0}^{1}(\mathcal{O})}\left\|U_{1}-U_{2}\right\|_{L^{2}(\mathcal{O})}\left\|U_{1}-U_{2}\right\|_{H_{0}^{1}(\mathcal{O})} \\
\leq & \frac{d_{1} \delta}{4}\left\|U_{1}-U_{2}\right\|_{H_{0}^{1}(\mathcal{O})}^{2}+\frac{2 M_{u}^{2} C_{N}^{4}}{d_{1} \delta}\left\|V_{1}-V_{2}\right\|_{H_{0}^{1}(\mathcal{O})}^{2} \\
& +\frac{2 \gamma^{2} C_{N}^{4}\left\|V_{2}\right\|_{H_{0}^{1}(\mathcal{O})}^{2}}{d_{1} \delta}\left\|U_{1}-U_{2}\right\|_{L^{2}(\mathcal{O})}^{2} .
\end{aligned}
$$

Combining the above inequality with (4.5) and (4.6), we obtain

$$
\frac{d}{d t}\left(\left\|U_{1}-U_{2}\right\|_{L^{2}(\mathcal{O})}^{2}+\frac{8 M_{u}^{2} C_{N}^{4}}{d_{1} d_{2} \delta^{2}}\left\|V_{1}-V_{2}\right\|_{L^{2}(\mathcal{O})}^{2}\right) \leq \bar{M}\left(\left\|U_{1}-U_{2}\right\|_{L^{2}(\mathcal{O})}^{2}+\left\|V_{1}-V_{2}\right\|_{L^{2}(\mathcal{O})}^{2}\right),
$$

where

$$
\bar{M}(t)=\frac{4 \gamma^{2} C_{N}^{4}\left\|V_{2}(t)\right\|_{H_{0}^{1}(\mathcal{O})}^{2}}{d_{1} \delta}+\frac{M_{b}^{2}}{d_{1} \delta}+\alpha+2+\left(\frac{M_{c}^{2}}{d_{2} \delta}+1\right) * \frac{8 M_{u}^{2} C_{N}^{4}}{d_{1} d_{2} \delta^{2}} .
$$

Recalling that $V_{1}, V_{2} \in L^{2}\left(0, T ; H_{0}^{1}(\mathcal{O})\right)$, so $\int_{0}^{T} \bar{M}(s) d t<\infty$. Thus we can obtain uniqueness immediately from the above inequality, the Gronwall inequality, and the fact $F_{1}-F_{2}=$ $U_{1}-U_{2}, G_{1}-G_{2}=V_{1}-V_{2}, u_{10}=u_{20}, v_{10}=v_{20}$.

Next, we will show the existence of a weak solution. Let $F_{0, m}, G_{0, m} \in H_{0}^{1}(\mathcal{O}), m=1,2, \ldots$, such that

$$
\begin{aligned}
& F_{0, m} \rightarrow F_{0} \quad \text { in } L^{2}(\mathcal{O}) \text {, as } m \rightarrow \infty, \\
& G_{0, m} \rightarrow G_{0} \quad \text { in } L^{2}(\mathcal{O}) \text {, as } m \rightarrow \infty .
\end{aligned}
$$

Then for each $F_{0, m}, G_{0, m}, m=1,2, \ldots$, there exists a unique strong solution $\left(F_{m}, G_{m}\right)$ for (3.1). We deduce from (3.16) and (3.17) that, for $\mathbb{P}$-a.s. $\omega \in \Omega$,

$$
\left\{F_{m}\right\} \text { is bounded in } C\left([0, T] ; L^{2}(\mathcal{O})\right) \cap L^{2}\left(0, T, H_{0}^{1}(\mathcal{O})\right) \cap L^{4}\left(0, T ; L^{4}(\mathcal{O})\right)
$$

and

$$
\left\{G_{m}\right\} \text { is bounded in } C\left([0, T] ; L^{2}(\mathcal{O})\right) \cap L^{2}\left(0, T, H_{0}^{1}(\mathcal{O})\right) \text {, }
$$

which implies that

$$
\text { the sequence }\left\{\gamma F_{m} G_{m}-F_{m}^{3}\right\} \text { is bounded in } L^{4 / 3}\left(0, T ; L^{4 / 3}(\mathcal{O})\right) \text {. }
$$

Therefore, we can extract a subsequence (denoted also by $\left\{\left(F_{m}, G_{m}\right)\right\}$ ) such that $\mathbb{P}$-a.s. $\omega \in$ $\Omega$

$$
\begin{aligned}
& F_{m} \rightarrow F \quad \text { weakly in } L^{2}\left(0, T ; H_{0}^{1}(\mathcal{O})\right) \\
& G_{m} \rightarrow G \quad \text { weakly in } L^{2}\left(0, T ; H_{0}^{1}(\mathcal{O})\right) \\
& \gamma F_{m} G_{m}-F_{m}^{3} \rightarrow \Phi \quad \text { weakly in } L^{4 / 3}\left(0, T ; L^{4 / 3}(\mathcal{O})\right) .
\end{aligned}
$$


Combining the arguments of the uniqueness and the fact (4.7), (4.8), which implies that $\left\{F_{m}\right\}$ and $\left\{G_{m}\right\}$ are Cauchy sequences in $C\left([0, T] ; L^{2}(\mathcal{O} \times \Omega)\right)$, the uniqueness of the limit and (4.12)-(4.13) yield, for $\mathbb{P}$-a.s. $\omega \in \Omega$,

$$
\begin{aligned}
& F_{m} \rightarrow F \quad \text { in } C\left([0, T] ; L^{2}(\mathcal{O})\right) \quad \text { and } \\
& G_{m} \rightarrow G \quad \text { in } C\left([0, T] ; L^{2}(\mathcal{O})\right), \quad \text { as } m \rightarrow \infty .
\end{aligned}
$$

Therefore, extracting a subsequence if necessary, we can assume that $\gamma F_{m} G_{m}-F_{m}^{3} \rightarrow$ $\gamma F G-F^{3}$, a.e. in $\mathcal{O} \times[0, T]$ as $m \rightarrow \infty$. Then (4.14) implies that $\Phi=\gamma F G-F^{3}$. Meanwhile, for any test function $\vartheta \in \mathcal{U}_{0, T},\left(F_{m}, G_{m}\right)$ satisfies (4.1) and (4.2). By using (4.12), (4.13), (4.14), and (4.15), and passing to the limit, we see that $(F, G)$ also satisfies (4.1) and (4.2). The estimates (4.3) and (4.4) can be obtained from (3.16), (3.17), (4.7), (4.8), and (4.15) directly. Thus, we can see $(F, G)$ is a weak solution of $(3.1)$ with initial $\left(u_{0}, v_{0}\right)$ by all arguments above. Then the proof of Theorem 4.1 is completed.

Remark 4.1 Since $(F, G) \in\left(L^{2}\left(0, T ; H_{0}^{1}(\mathcal{O})\right)\right)^{2}$, for any $t \in(\tau, T)$, $\mathbb{P}$-a.s. $\omega \in \Omega$, it follows that there exists an earlier time $t_{0} \in(0, t)$ satisfying that $(F, G) \in\left(H_{0}^{1}(\mathcal{O})\right)^{2}$, which implies that the weak solutions of (3.1) turn into the strong solutions after a null measure set $\left(\tau, t_{0}\right)$. Hence, we obtain $\left(F^{\prime}, G^{\prime}\right) \in L^{2}\left(0, T ; L^{2}(\mathcal{O})\right) \times L^{2}\left(0, T ; L^{2}(\mathcal{O})\right)$ and $(F, G) \in C\left(0, T ; L^{2}(\mathcal{O})\right) \times$ $C\left(0, T ; L^{2}(\mathcal{O})\right)$.

Definition 4.2 A function $(F, G): \bigcup_{t \in[0, \infty)} \mathcal{O} \times t \rightarrow \mathbb{R}^{2}$ is called a weak solution of (2.13) if for any $T \geq 0$, the restriction of $(F, G)$ on $\bigcup_{t \in[0, T)} \mathcal{O} \times t$ is a weak solution of (3.1).

Repeating arguments similar to Theorem 4.1, we obtain the following result.

Theorem 4.2 Under the same assumptions of Theorem 3.1, for any $\left(u_{0}, v_{0}\right) \in L^{2}\left(\mathcal{O}_{0}\right) \times$ $L^{2}\left(\mathcal{O}_{0}\right),(2.13)$ has a unique weak solution.

\section{The non-autonomous pullback $\mathfrak{D}_{\sigma}$-attractor for SBS}

In this section, we will establish some priori estimates for the solutions of (2.13), and introduce the 'partial-random' dynamical system generated by weak solution. By following the argument in [8], we prove the existence of the non-autonomous pullback $\mathscr{D}_{\sigma}$-attractor for the system.

Assume that $(F, G)$ is a weak solution of $(2.13)$ with initial value $\left(F_{0}, G_{0}\right)$. Let

$$
\begin{aligned}
& \bar{M}_{a}=N \max _{1 \leq j, k \leq N}\left\|a_{j k}\right\|_{L^{\infty}(\mathcal{O} \times \mathbb{R})}, \\
& \bar{M}_{b}=N^{1 / 2} \max _{1 \leq k \leq N}\left\|b_{k}\right\|_{L^{\infty}(\mathcal{O} \times \mathbb{R})}, \quad \bar{M}_{c}=N^{1 / 2} \max _{1 \leq k \leq N}\left\|c_{k}\right\|_{L^{\infty}(\mathcal{O} \times \mathbb{R})},
\end{aligned}
$$

and

$$
\bar{M}_{\bar{b}}=N^{1 / 2} \max _{1 \leq k \leq N}\left\|\bar{b}_{k}\right\|_{L^{\infty}(\mathcal{O} \times \mathbb{R})}, \quad \bar{M}_{\bar{c}}=N^{1 / 2} \max _{1 \leq k \leq N}\left\|\bar{c}_{k}\right\|_{L^{\infty}(\mathcal{O} \times \mathbb{R})}
$$

where $\bar{b}, \bar{c}$ are defined in the proof of Theorem 3.1. We will also assume that $\bar{M}_{a}<\infty$, $\bar{M}_{b}<\infty, \bar{M}_{c}<\infty, \bar{M}_{\bar{b}}<\infty$, and $\bar{M}_{\bar{c}}<\infty$. 
Lemma 5.1 There exist two positive constants $M, C$, and a random process $R_{4}$ such that for $\mathbb{P}$-a.s. $\omega \in \Omega, t \geq \tau$

$$
\begin{aligned}
& \|F(t)\|_{L^{2}(\mathcal{O})}^{2}+\|G(t)\|_{L^{2}(\mathcal{O})}^{2} \\
& \quad \leq M e^{-C(t-\tau)}\left(\|F(\tau)\|_{L^{2}(\mathcal{O})}^{2}+\|G(\tau)\|_{L^{2}(\mathcal{O})}^{2}\right)+\int_{\tau}^{t} e^{-C(t-s)} R_{4}(s, \omega) d s .
\end{aligned}
$$

Proof Taking the inner product of the first formula of (2.13) with $F$ and the second formula with $G$ in $L^{2}(\mathcal{O})$, then using Cauchy's inequality, Hölder's inequality, and Lemma 3.1, we can obtain

$$
\begin{aligned}
& \frac{d}{d t}\|F(t)\|_{L^{2}(\mathcal{O})}^{2}+d_{1} \delta\|F(t)\|_{H_{0}^{1}(\mathcal{O})}^{2}+\|F(t)\|_{L^{4}(\mathcal{O})}^{4} \\
& \quad \leq M_{1}\|F(t)\|_{L^{2}(\mathcal{O})}^{2}+M_{2}\|G(t)\|_{L^{2}(\mathcal{O})}^{2}+R_{5}(t, \omega)
\end{aligned}
$$

where $M_{1}=\frac{2 \bar{M}_{b}^{2}}{d_{1} \delta}+\bar{M}_{b}+2 \alpha+\gamma+4, M_{2}=\alpha+5 \gamma^{2}$, and

$$
\begin{aligned}
R_{5}(t, \omega)= & \left(\frac{2 d_{1} \bar{M}_{a}^{2}}{\delta}+\bar{M}_{b}\right)\left\|W_{1}\right\|_{H_{0}^{1}(\mathcal{O})}^{2}+\left\|W_{1}\right\|_{L^{2}(\mathcal{O})}^{2}+\left(\alpha+4 \gamma^{2}\right)\left\|W_{2}\right\|_{L^{2}(\mathcal{O})}^{2} \\
& +\left(\frac{\gamma}{2}+709\right)\left\|W_{1}\right\|_{L^{4}(\mathcal{O})}^{4}+\frac{\gamma}{2}\left\|W_{2}\right\|_{L^{4}(\mathcal{O})}^{4}+\left\|R_{1}\right\|_{L^{2}(\mathcal{O})}^{2} .
\end{aligned}
$$

Similarly, we have

$$
\frac{d}{d t}\|G(t)\|_{L^{2}(\mathcal{O})}^{2}+d_{2} \delta\|G(t)\|_{H_{0}^{1}(\mathcal{O})}^{2} \leq\|F(t)\|_{L^{2}(\mathcal{O})}^{2}+M_{3}\|G(t)\|_{L^{2}(\mathcal{O})}^{2}+R_{6}(t, \omega),
$$

where $M_{3}=\frac{2 \bar{M}_{c}^{2}}{d_{2} \delta}+\bar{M}_{c}+4-\beta$, and

$$
R_{6}(t, \omega)=\left(\frac{2 d_{2} \bar{M}_{a}^{2}}{\delta}+\bar{M}_{c}\right)\left\|W_{1}\right\|_{H_{0}^{1}(\mathcal{O})}^{2}+\left\|W_{1}\right\|_{L^{2}(\mathcal{O})}^{2}+\beta\left\|W_{2}\right\|_{L^{2}(\mathcal{O})}^{2}+\left\|R_{2}\right\|_{L^{2}(\mathcal{O})}^{2}
$$

Choosing $\beta>\max _{\delta}\left\{\frac{2 \bar{M}_{c}^{2}}{d_{2} \delta}+\bar{M}_{c}+4\right\}$ such that $M_{3}<0$, and denoting $\tilde{M}_{3}=-M_{3}$. We can derive from (5.5) and (5.6) that

$$
\begin{aligned}
& \frac{d}{d t}\left(\|F(t)\|_{L^{2}(\mathcal{O})}^{2}+\bar{C}\|G(t)\|_{L^{2}(\mathcal{O})}^{2}\right)+d_{1} \delta\|F(t)\|_{H_{0}^{1}(\mathcal{O})}^{2}+\bar{C} d_{2} \delta\|G(t)\|_{H_{0}^{1}(\mathcal{O})}^{2}+\|F(t)\|_{L^{4}(\mathcal{O})}^{4} \\
& \quad \leq\left(M_{1}+\bar{C}\right)\|F(t)\|_{L^{2}(\mathcal{O})}^{2}+\left(M_{2}-\bar{C} \tilde{M}_{3}\right)\|G(t)\|_{L^{2}(\mathcal{O})}^{2}+R_{5}(t, \omega)+\bar{C} R_{6}(t, \omega)
\end{aligned}
$$

Let $\bar{C}=\frac{2 M_{2}}{\tilde{M}_{3}}$, then

$$
\begin{aligned}
& \frac{d}{d t}\left(\|F(t)\|_{L^{2}(\mathcal{O})}^{2}+\bar{C}\|G(t)\|_{L^{2}(\mathcal{O})}^{2}\right)+d_{1} \delta\|F(t)\|_{H_{0}^{1}(\mathcal{O})}^{2}+\bar{C} d_{2} \delta\|G(t)\|_{H_{0}^{1}(\mathcal{O})}^{2} \\
& \quad+\frac{M_{2}}{\bar{C}}\|F(t)\|_{L^{2}(\mathcal{O})}^{2}+M_{2}\|G(t)\|_{L^{2}(\mathcal{O})}^{2}+\frac{1}{4}\|F(t)\|_{L^{4}(\mathcal{O})}^{4} \\
& \leq \frac{d}{d t}\left(\|F(t)\|_{L^{2}(\mathcal{O})}^{2}+\bar{C}\|G(t)\|_{L^{2}(\mathcal{O})}^{2}\right)+d_{1} \delta\|F(t)\|_{H_{0}^{1}(\mathcal{O})}^{2}+\bar{C} d_{2} \delta\|G(t)\|_{H_{0}^{1}(\mathcal{O})}^{2}
\end{aligned}
$$




$$
\begin{gathered}
+\frac{1}{2}\|F(t)\|_{L^{4}(\mathcal{O})}^{4}+M_{2}\|G(t)\|_{L^{2}(\mathcal{O})}^{2}+\frac{M_{2}^{2}}{\bar{C}^{2}}|\mathcal{O}| \\
\leq\left(\frac{\left(M_{1}+\bar{C}\right)^{2}}{2}+\frac{M_{2}^{2}}{\bar{C}^{2}}\right)|\mathcal{O}|+R_{5}(t, \omega)+\bar{C} R_{6}(t, \omega) .
\end{gathered}
$$

Denote

$$
R_{7}(t, \omega)=\left(\frac{\left(M_{1}+\bar{C}\right)^{2}}{2}+\frac{M_{2}^{2}}{\bar{C}^{2}}\right)|\mathcal{O}|+R_{5}(t, \omega)+\bar{C} R_{6}(t, \omega),
$$

the inequality (5.8) implies that

$$
\begin{aligned}
& \frac{d}{d t}\left(\|F(t)\|_{L^{2}(\mathcal{O})}^{2}+\bar{C}\|G(t)\|_{L^{2}(\mathcal{O})}^{2}\right)+\frac{M_{2}}{\bar{C}}\left(\|F(t)\|_{L^{2}(\mathcal{O})}^{2}+\bar{C}\|G(t)\|_{L^{2}(\mathcal{O})}^{2}\right) \\
& \quad+d_{1} \delta\|F(t)\|_{H_{0}^{1}(\mathcal{O})}^{2}+\bar{C} d_{2} \delta\|G(t)\|_{H_{0}^{1}(\mathcal{O})}^{2}+\frac{1}{4}\|F(t)\|_{L^{4}(\mathcal{O})}^{4} \leq R_{7}(t, \omega) .
\end{aligned}
$$

From the Gronwall inequality, we see that for $\mathbb{P}$-a.s. $\omega \in \Omega$

$$
\begin{aligned}
& \|F(t)\|_{L^{2}(\mathcal{O})}^{2}+\bar{C}\|G(t)\|_{L^{2}(\mathcal{O})}^{2} \\
& \quad \leq e^{-\frac{M_{2}}{\bar{C}}(t-\tau)}\left(\|F(\tau)\|_{L^{2}(\mathcal{O})}^{2}+\bar{C}\|G(\tau)\|_{L^{2}(\mathcal{O})}^{2}\right)+\int_{\tau}^{t} e^{-\frac{M_{2}}{\bar{C}(s-\tau)}} R_{7}(s, \omega) d s .
\end{aligned}
$$

Denoting $M=\frac{\max \{1, \bar{C}\}}{\min \{1, \bar{C}\}}, C=\frac{M_{2}}{\bar{C}}$ and $R_{4}(t, \omega)=\frac{1}{\min \{1, \bar{C}\}} R_{7}(t, \omega)$, the proof is completed.

Lemma 5.2 For any nonrandom bounded set $B \in L^{2}(\mathcal{O}) \times L^{2}(\mathcal{O})$, there exists a random time $T_{B}(\omega) \geq 0$ such that

$$
\|F(t)\|_{L^{2}(\mathcal{O})}^{2}+\|G(t)\|_{L^{2}(\mathcal{O})}^{2} \leq 2 \int_{-\infty}^{t} e^{-C(t-s)} R_{4}(s, \omega) d s
$$

for $\mathbb{P}$-a.s. $\omega \in \Omega$, for all $t-\tau \geq T_{b}(\omega)$, for any $(F(\tau), G(\tau)) \in B$.

Proof It follows from Lemma 5.1 that

$$
\begin{aligned}
& \|F(t)\|_{L^{2}(\mathcal{O})}^{2}+\|G(t)\|_{L^{2}(\mathcal{O})}^{2} \\
& \quad \leq M e^{-C(t-\tau)}\left(\|F(\tau)\|_{L^{2}(\mathcal{O})}^{2}+\|G(\tau)\|_{L^{2}(\mathcal{O})}^{2}\right)+\int_{\tau}^{t} e^{-C(t-s)} R_{4}(s, \omega) d s .
\end{aligned}
$$

We can obtain from the above inequality that

$$
e^{-C(t-\tau)}\left(\|F(\tau)\|_{L^{2}(\mathcal{O})}^{2}+\|G(\tau)\|_{L^{2}(\mathcal{O})}^{2}\right) \rightarrow 0 \quad \text { as } t-\tau \rightarrow \infty
$$

Then there exists a random time $T_{B}(\omega)$ such that for $t-\tau \geq T_{B}(\omega)$

$$
e^{-C(t-\tau)}\left(\|F(\tau)\|_{L^{2}(\mathcal{O})}^{2}+\|G(\tau)\|_{L^{2}(\mathcal{O})}^{2}\right) \leq \int_{-\infty}^{t} e^{-C(t-s)} R_{4}(s, \omega) d s
$$

Thus, the proof is completed. 
Corollary 5.1 For any nonrandom bounded $B \in L^{2}(\mathcal{O}) \times L^{2}(\mathcal{O})$, there exist a random time $T_{B}(\omega) \geq 0$ such that

$$
\int_{t}^{t+1}\|F(s)\|_{H_{0}^{1}(\mathcal{O})}^{2}+\|G(s)\|_{H_{0}^{1}}^{2}+\|F(t)\|_{L^{4}(\mathcal{O})}^{4} d s \leq \hat{M}
$$

for $\mathbb{P}$-a.s. $\omega \in \Omega$, for all $t-\tau \geq T_{B}(\omega)$, for any $(F(\tau), G(\tau)) \in B$.

Lemma 5.3 For any nonrandom bounded $B \in L^{2}(\mathcal{O}) \times L^{2}(\mathcal{O})$, there exists a random time $\bar{T}_{B}(\omega) \geq 0$ and a random constant $\tilde{M}$, such that

$$
\|F(s)\|_{H_{0}^{1}(\mathcal{O})}^{2}+\|G(s)\|_{H_{0}^{1}(\mathcal{O})}^{2} \leq \tilde{M}
$$

for $\mathbb{P}$-a.s. $\omega \in \Omega$, for all $t-\tau \geq T_{B}(\omega)$, for any $(F(\tau), G(\tau)) \in B$.

Proof Taking the inner product of the second formula in (2.13) with $-\triangle G$ in $L^{2}(\mathcal{O})$, and using Cauchy's inequality, Hölder's inequality, and Lemma 3.2, we obtain

$$
\begin{aligned}
& \frac{d}{d t}\|G(t)\|_{H_{0}^{1}(\mathcal{O})}^{2}+d_{2} \delta_{0}\|\Delta G(t)\|_{L^{2}(\mathcal{O})}^{2} \\
& \leq\left(\frac{4 \bar{M}_{\bar{c}}^{2}}{d_{2} \delta_{0}}-\beta\right)\|G(t)\|_{H_{0}^{1}(\mathcal{O})}^{2}+2 d_{2} c_{0}\|G(t)\|_{L^{2}(\mathcal{O})}^{2}+\frac{4 d_{2} \bar{M}_{a}^{2}}{\delta_{0}}\left\|\Delta W_{2}\right\|_{L^{2}(\mathcal{O})}^{2} \\
& \quad+\left(\frac{4 \bar{M}_{\bar{c}}^{2}}{d_{2} \delta_{0}}+\beta\right)\left\|W_{2}(t)\right\|_{H_{0}^{1}(\mathcal{O})}^{2}+\frac{3}{d_{2} \delta_{0}}\left(\|F\|_{L^{2}(\mathcal{O})}^{2}+\left\|W_{1}\right\|_{L^{2}(\mathcal{O})}^{2}+\left\|R_{2}\right\|_{L^{2}(\mathcal{O})}^{2}\right)
\end{aligned}
$$

Combining the assumptions (2.5), (2.14) with Lemmas 5.1-5.2, we have

$$
\begin{aligned}
& \int_{t}^{t+1} 2 d_{2} c_{0}\|G(s)\|_{L^{2}(\mathcal{O})}^{2}+\frac{4 d_{2} \bar{M}_{a}^{2}}{\delta_{0}}\left\|\Delta W_{2}\right\|_{L^{2}(\mathcal{O})}^{2}+\left(\frac{4 \bar{M}_{\bar{c}}^{2}}{d_{2} \delta_{0}}+\beta\right)\left\|W_{2}(s)\right\|_{H_{0}^{1}(\mathcal{O})}^{2} \\
& +\frac{3}{d_{2} \delta_{0}}\left(\|F(s)\|_{L^{2}(\mathcal{O})}^{2}+\left\|W_{1}\right\|_{L^{2}(\mathcal{O})}^{2}+\left\|R_{2}\right\|_{L^{2}(\mathcal{O})}^{2}\right) d s<\infty
\end{aligned}
$$

for $\mathbb{P}$-a.s. $\omega \in \Omega$ and for all $t-\tau>T_{B}(\omega)$. Therefore there exists a constant $\tilde{M}$ such that

$$
\|G\|_{H_{0}^{1}(\mathcal{O})}^{2} \leq \tilde{M}
$$

for $\mathbb{P}$-a.s. $\omega \in \Omega$ and for all $t-\tau>T_{B}(\omega)+1$.

Similarly,

$$
\begin{aligned}
\frac{d}{d t}\|F\|_{H^{1}(\mathcal{O})}^{2}+d_{1} \delta_{0}\|\Delta F\|_{L^{2}(\mathcal{O})}^{2} \\
\leq\left(\frac{4 \bar{M}_{\bar{b}}^{2}}{d_{1} \delta_{0}}+\alpha+3\right)\|F\|_{H_{0}^{1}(\mathcal{O})}^{2}+2 d_{1} C_{0}\|F\|_{L^{2}(\mathcal{O})}^{2}+\frac{4 d_{1} \bar{M}_{a}^{2}}{\delta_{0}}\left\|\Delta W_{1}\right\|_{L^{2}(\mathcal{O})}^{2} \\
\quad+\left(\frac{4 \bar{M}_{\bar{b}}^{2}}{d_{1} \delta_{0}}+1\right)\left\|W_{1}\right\|_{H_{0}^{1}(\mathcal{O})}^{2}+\alpha\left\|G+W_{2}\right\|_{H_{0}^{1}(\mathcal{O})}^{2}+\left(\frac{2 \gamma^{2}}{d_{1} \delta_{0}}+\frac{3}{2}\right)\left\|F+W_{1}\right\|_{L^{4}(\mathcal{O})}^{4} \\
\quad+\frac{2 \gamma^{2}}{d_{1} \delta_{0}}\left\|G+W_{2}\right\|_{L^{4}(\mathcal{O})}^{4}+\frac{1}{2}\left\|\Delta W_{1}\right\|_{L^{4}(\mathcal{O})}^{4}
\end{aligned}
$$


Due to (2.5), (2.14), we have

$$
\begin{aligned}
& \int_{t}^{t+1} 2 d_{1} C_{0}\|F\|_{L^{2}(\mathcal{O})}^{2}+\frac{4 d_{1} \bar{M}_{a}^{2}}{\delta_{0}}\left\|\Delta W_{1}\right\|_{L^{2}(\mathcal{O})}^{2} \\
& +\left(\frac{4 \bar{M}_{\bar{b}}^{2}}{d_{1} \delta_{0}}+1\right)\left\|W_{1}\right\|_{H_{0}^{1}(\mathcal{O})}^{2}+\alpha\left\|G+W_{2}\right\|_{H_{0}^{1}(\mathcal{O})}^{2} \\
& +\left(\frac{2 \gamma^{2}}{d_{1} \delta_{0}}+\frac{3}{2}\right)\left\|F+W_{1}\right\|_{L^{4}(\mathcal{O})}^{4}+\frac{2 \gamma^{2}}{d_{1} \delta_{0}}\left\|G+W_{2}\right\|_{L^{4}(\mathcal{O})}^{4} \\
& \quad+\frac{1}{2}\left\|\triangle W_{1}\right\|_{L^{4}(\mathcal{O})}^{4} d s<\infty,
\end{aligned}
$$

for $\mathbb{P}$-a.s. $\omega \in \Omega$ and for all $t-\tau>T_{B}(\omega)+1$. Thus, applying the uniform Gronwall lemma to (5.16), we see that there exists a constant $\tilde{M}$ such that

$$
\|F\|_{H_{0}^{1}(\mathcal{O})}^{2} \leq \tilde{M}
$$

for $\mathbb{P}$-a.s. $\omega \in \Omega$ and for all $t-\tau>T_{B}(\omega)+2$. Denoting $\bar{T}_{B}(\omega)=T_{B}(\omega)+2$, we complete the proof.

\section{Attractors for partial-random dynamical system}

In this section, we introduce the partial-random dynamical system generated by a SPDE defined on time-varying domains developed by Crauel et al. in [4], and prove the existence of the non-autonomous attractor for partial-random dynamical system.

Assume that the probability space $(\Omega, \mathcal{F}, \mathbb{P})$ with incremental shifts $\left(\kappa_{t}\right)_{t \in \mathbb{R}}$ is a metric dynamical system, $\Re$ is a subset of the topology of space $C_{b}^{1}\left(\mathbb{R} ; C_{b}^{2}\left(\overline{\mathcal{O}} ; \mathbb{R}^{N}\right)\right)$ generated by the domain varying diffeomorphisms $r$. The transformations $\pi_{t}: \mathfrak{R} \rightarrow \mathfrak{R}$ defined by $\pi_{t} r(\cdot+$ $s, \cdot)=r(\cdot+s+t, \cdot)$ for $t \in \mathbb{R}$, form a one-parameter group $\left(\pi_{t}\right)_{t \in \mathbb{R}}$ with

$$
\pi_{t+s}=\pi_{t} \circ \pi_{s}
$$

for all $s, t \in \mathbb{R}$. The product flow, given by

$$
(\kappa \times \pi)_{t}=\kappa_{t} \times \pi_{t}: \omega \times \mathfrak{R} \rightarrow \omega \times \mathfrak{R}
$$

for $t \in \mathbb{R}$, will be denoted by $\left(\bar{\kappa}_{t}\right)_{t \in \mathbb{R}}$.

For each $\left(F_{0}, G_{0}\right) \in\left(L^{2}(\mathcal{O})\right)^{2}$, Theorem 4.2 implies that equations (2.13) have a unique global solution $(F, G)$. Define the operators

$$
\Upsilon(t,(\omega, r)): L^{2}(\mathcal{O}) \times L^{2}(\mathcal{O}) \rightarrow L^{2}(\mathcal{O}) \times L^{2}(\mathcal{O})
$$

by

$$
\Upsilon(t,(\omega, r))\left(F_{0}, G_{0}\right)=\left(F\left(t ;(\omega, r), F_{0}, G_{0}\right), G\left(t ;(\omega, r), F_{0}, G_{0}\right)\right)=(F(t), G(t)) .
$$

Here $\left(F\left(t ;(\omega, r), F_{0}, G_{0}\right), G\left(t ;(\omega, r), F_{0}, G_{0}\right)\right)$ is defined by unique solution process of (5.9) with initial value $\left(F_{0}, G_{0}\right)$ and the transform for domains $r$. From Theorem 4.2, we know 
that the definition makes sense. Then the family of operators $\{\Upsilon(t): 0 \leq t<+\infty\}$ generates a non-autonomous dynamic system, i.e.

$$
\begin{aligned}
& \Upsilon(0,(\omega, r))=\operatorname{Id}\left(\text { identity on } L^{2}(\mathcal{O})\right) \quad \forall(\omega, r) \in \Omega \times \Re, \\
& \Upsilon(t+s,(\omega, r)) \\
& \quad=\Upsilon\left(t, \bar{\kappa}_{s} p(\omega, r)\right) \circ \Upsilon(s,(\omega, r)) \quad \text { for all } s, t \in[0, \infty) \text { and }(\omega, r) \in \Omega \times \Re .
\end{aligned}
$$

Now, we can define the attractor of the non-autonomous dynamic system $\Upsilon$.

Definition 6.1 ([4]) Suppose that $\mathcal{D}$ is a set of maps from $\Omega \times \Re$ to the power set of $L^{2}(\mathcal{O}) \times L^{2}(\mathcal{O})$ such that $D(\omega, r)$ is nonempty for every $(\omega, r) \in \Omega \times \Re$ and $D \in \mathcal{D}$. A map $A$ from $\Omega \times \mathfrak{R}$ to the power set of $L^{2}(\mathcal{O}) \times L^{2}(\mathcal{O})$ is said to be a $\mathcal{D}$-attractor if:

(1) $A(\omega, r)$ is compact for all $(\omega, r) \in \Omega \times \Re$,

(2) $A$ is invariant in the sense that

$$
\Upsilon(t,(\omega, r)) A(\omega, r)=\bar{\kappa}_{t} A(\omega, r)
$$

for all $t \in[0, \infty)$ and $(\omega, r) \in \Omega \times \Re$,

(3) $A$ attracts every $D \in \mathcal{D}$ in the sense that

$$
\lim _{t \rightarrow \infty} \operatorname{dist}\left(\Upsilon\left(t, \bar{\kappa}_{-t}(\omega, r)\right) D\left(\bar{\kappa}_{-t}(\omega, r)\right), A(\omega, r)\right)=0
$$

for every $D \in \mathcal{D}$.

Here $\operatorname{dist}(A, D)$ is for the Hausdorff semi-distance.

Definition 6.2 ([4]) Suppose that $\mathcal{D}$ is a set of maps from $\Omega \times \mathfrak{R}$ to the power set of $L^{2}(\mathcal{O}) \times L^{2}(\mathcal{O})$ such that $D(\omega, r)$ is nonempty for every $(\omega, r) \in \Omega \times \Re$ and $D \in \mathcal{D}$. A map $K$ from $\Omega \times \Re$ to the power set of $L^{2}(\mathcal{O}) \times L^{2}(\mathcal{O})$ is said to be a $\mathcal{D}$-attracting if

$$
\lim _{t \rightarrow \infty} \operatorname{dist}\left(\Upsilon\left(t, \bar{\kappa}_{-t}(\omega, r)\right) D\left(\bar{\kappa}_{-t}(\omega, r)\right), K(\omega, r)\right)=0
$$

for every $D \in \mathcal{D}$.

Theorem 6.1 ([4]) The existence of a compact $\mathcal{D}$-attracting $K$ is equivalent to the existence of a $\mathcal{D}$-attractor.

Remark 6.1 From Lemmas 5.2 and 5.3, we can find that there exists a compact $\mathcal{D}$ attracting $K$ for the non-autonomous dynamic system $\Upsilon$ defined above, attracting bounded subsets of $L^{2}(\mathcal{O}) \times L^{2}(\mathcal{O})$. Thus, using Theorem 6.1, we can obtain a unique non-autonomous pullback attractor in $L^{2}(\mathcal{O}) \times L^{2}(\mathcal{O})$.

Theorem 6.2 The partial-random system generated by the random-PDE (2.13) on domain $\mathcal{O}$ has a unique non-autonomous pullback attractor in $L^{2}(\mathcal{O}) \times L^{2}(\mathcal{O})$, attracting bounded subsets of $L^{2}(\mathcal{O}) \times L^{2}(\mathcal{O})$. 
Authors' contributions

All authors read and approved the final manuscript.

\section{Acknowledgements}

The authors would like to thank the anonymous referees and editors for their helpful suggestions, which have improved the quality of the paper largely. This work was supported by the NSF of China (No.11371367, 11571126).

Received: 13 March 2016 Accepted: 10 May 2016 Published online: 20 May 2016

\section{References}

1. Arnold, L: Random Dynamical Systems. Springer, Berlin (1998)

2. Bonaccorsi, S, Guatteri, G: A variational approach to evolution problems with variable domains. J. Differ. Equ. 175 51-70 (2001)

3. Crauel, H, Debussche, A, Flandoli, F: Random attractors. J. Differ. Equ. 9, 307-341 (1997)

4. Crauel, H, Kloeden, P, Yang, M: Random attractors of stochastic reaction-diffusion equations on variable domains. Stoch. Dyn. 11, 301-314 (2011)

5. He, C, Hsiao, L: Two-dimensional Euler equations in a time dependent domain. J. Differ. Equ. 163, $265-291$ (2000)

6. Kloeden, P, Real, J, Sun, C: Pullback attractors for a semilinear heat equation on time-varying domains. J. Differ. Equ. 246, 4702-4730 (2009)

7. Kloeden, P, Maín-Rubio, P, Real, J: Pullback attractors for a semilinear heat equation in a non-cylindrical domain. J. Differ. Equ. 244, 2062-2090 (2008)

8. Kloeden, P, Rasmussen, M: Nonautonomous Dynamical Systems. Am. Math. Soc., Providence (2011)

9. Límaco, J, Medeiros, LA, Zuazua, E: Existence, uniqueness and controllability for parabolic equations in non-cylindrical domains. Mat. Contemp. 23, 49-70 (2002)

10. Sun, $C$, Yuan, $Y: L^{p}$-Type pullback attractors for a semilinear heat equation on time-varying domains. Proc. R. Soc. Edinb., Sect. A, Math. 145(5), 1029-1052 (2015)

11. Crauel, H, Kloeden, P, Real, J: Stochastic partial differential equations with additive noise on time-varying domains. Bol. Soc. Esp. Mat. Apl. 51, 41-48 (2010)

12. Dufiet, V, Boissonade, J: Dynamics of Turing pattern monolayers close to onset. Phys. Rev. E 53, 4883-4892 (1996)

13. FitzHugh, R: Impulses and physiological states in theoretical models of nerve membrane. Biophys. J. 1, 445-466 (1961)

14. Nagumo, J, Arimoto, S, Yosimzawa, S: An active pulse transmission line simulating nerve axon. Proc. IRE 50, 2061-2070 (1964)

15. Tu, J: Global attractors and robustness of the Boissonade system. J. Dyn. Differ. Equ. 27, 187-211 (2015)

16. Evans, L: Partial Differential Equations. Grad. Stud. Math., vol. 19. Am. Math. Soc., Providence, RI (1998)

17. Lions, J: Quelques Méthodes de Résolution des Problèmes aux Limites Non Linéaires. Dunod, Paris (1969)

18. Robinson, J: Infinite-Dimensional Dynamical Systems. Cambridge University Press, Cambridge (2001)

\section{Submit your manuscript to a SpringerOpen ${ }^{\circ}$ journal and benefit from:}

- Convenient online submission

Rigorous peer review

- Immediate publication on acceptance

Open access: articles freely available online

- High visibility within the field

- Retaining the copyright to your article 